\title{
QUALITATIVE ANALYSIS ON A CHEMOTACTIC DIFFUSION MODEL FOR TWO SPECIES COMPETING FOR A LIMITED RESOURCE
}

BY

XUEFENG WANG (Department of Mathematics, Tulane University, New Orleans, LA)

AND

YAPING WU (Department of Mathematics, Capital Normal University, Beijing 100037, P.R. China)

1. Introduction and description of main results. We are interested in the effects of diffusivity and chemotaxis on the competition of several species for a limited resource. Diffusivity of cells is also called motility in some engineering literature. Chemotaxis is the oriented movement of cells in response to the concentration gradient of chemical substances in their environment. It is "anti-diffusion". It was observed experimentally and numerically (see [LAK, LC] and the references therein) that motility and chemotaxis of cells play a dominant role in the cell growth: when several species of cells compete for a limited resource, the species with smaller diffusion rate and larger chemotaxis rate enjoys better growth, even when the other species have superior growth kinetics.

To elucidate this effect of cell motility and chemotaxis on population growth, Lauffenburger, Aris and Keller [LAK] proposed a model of a single bacterial population in a 1-dimensional medium of finite length with growth limited by a nutrient diffusing from an adjacent phase not accessible to the bacteria. Their model is (in the dimensionless form):

$$
\left\{\begin{array}{lr}
u_{t}=u_{x x}-f(u) v, & 0<x<1, t>0 \\
v_{t}=\left(\lambda v_{x}-\chi v \phi^{\prime}(u) u_{x}\right)_{x}+(k f(u)-\theta) v, & 0<x<1, t>0 \\
u_{x}(0, t)=0, \quad u_{x}(1, t)=h(1-u(1, t)), & t>0 \\
\lambda v_{x}-\chi v \phi^{\prime}(u) u_{x}=0, & x=0,1, \quad t>0 .
\end{array}\right.
$$

Here $u$ is the concentration of the nutrient and $v$ the density of the bacteria, $f(u)$ is the consumption rate of the nutrient per cell; the term $(k f(u)-\theta) v$ in the $v$-equation represents that the bacteria have a Malthusian growth with $k f(u)$ and $\theta$ measuring the respective birth and death rates. $-u_{x}$ and $-\lambda v_{x}$ are the random flux of $u$ and $v$ respectively, while $\chi v \phi^{\prime}(u) u_{x}$ is the chemotactic flux of $v$, where $\lambda>0$ and $\chi \geq 0$ are constants; the total flux of $v$ at the boundary points $x=0$ and 1 is zero; this is true for

Received September 14, 2000.

2000 Mathematics Subject Classification. Primary 92C17. 
$u$ at $x=0$, but at $x=1, u$ is diffused into the medium. In the adjacent phase (which is the interval $(1, \infty)), u \equiv 1$, which must also be an upper bound for $u$ inside the medium, and therefore we are interested only in solutions with $0 \leq u \leq 1$.

From biological and technical considerations, we require $f$ and $\phi$ satisfying

$$
\begin{array}{cll}
f(0)=0, \quad f^{\prime}(u)>0 & \text { and } & \phi^{\prime}(u)>0 \text { on }[0, \infty), \\
f \in C^{3}([0, \infty)) & \text { and } \phi \in C^{5}([0, \infty)) .
\end{array}
$$

In [LAK], $\phi(u)$ is taken to be $u$. Numerical calculations on steady states of (1.1) (with $\phi(u)=u, \chi$ proportional to $\lambda$ ) led the authors of [LAK] to the following observations: (i) random motility $\lambda$ leads to decreased cell population $\int_{0}^{1} v(x) d x$. (ii) chemotaxis coefficient $\chi$ acts to increase $\int_{0}^{1} v(x) d x$.

Zeng $[\mathrm{Z}]$ studied the existence of positive steady states of (1.1), proving that they exist if and only if $0<\theta<k f(1)$. Wang [W] (i) investigated the effects of large or small $\lambda$ or $\chi$ on these positive steady states, supporting and adding to the observations in [LAK]; (ii) did so when the bacteria have a logistic growth type (which was not considered in $[\mathrm{LAK}]$ ), discovering that large $\chi$ drives the population to extinction; (iii) studied the stability of steady states and boundedness of global solutions.

In this paper, we consider the situation of two species of bacteria competing for the same nutrient, where the growth kinetics of both species are identical but their motility and chemotaxis coefficients are different. The interest is in the possibility of "competition exclusion" and stable coexistence, attributable solely to motility and chemotaxis. Let the competing species have density function $w$, and to focus solely on the effect of motility and chemotaxis, we assume that both species have the same consumption rate of the substrate, and the same birth and death rates. The model is

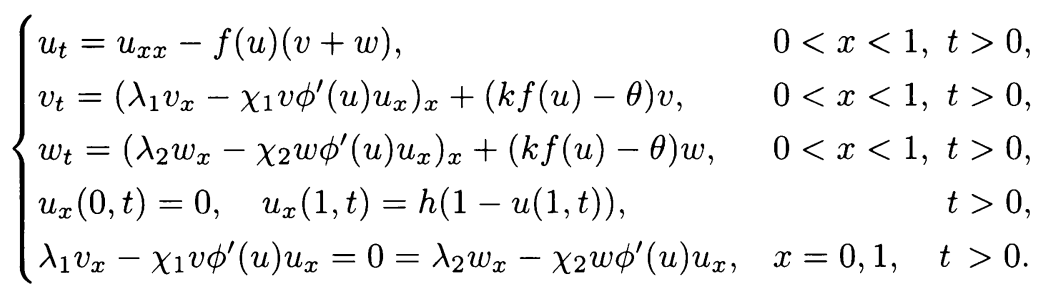

The existence, uniqueness, and boundedness of global-in-time solutions of (1.3) can be established as in the case of (1.1). This is also true for the stability/instability of the trivial steady state $(1,0,0)$ : it is globally asymptotically stable if $\theta \geq k f(1)$, and unstable if $0<\theta<k f(1)$. In particular, if $\theta \geq k f(1)$, the only nonnegative steady state of (1.3) is the trivial one. See Theorems 2.1 and 2.2 for the precise statements.

Now fix $\theta \in(0, k f(1))$. Then the existence result [Z] yields two semitrivial steady states $(\underline{u}(x), 0, \underline{w}(x))$ and $(\bar{u}(x), \bar{v}(x), 0)$. Our next set of results aims at giving the ranges for the motility and chemotaxis parameters $\lambda_{1}, \lambda_{2}, \chi_{1}$, and $\chi_{2}$ so that one species can wipe out the other, or they coexist in a stable equilibrium. In loose terms, they may be summarized as follows:

Let $\lambda_{1}>0$ and $\chi_{1} \geq 0$ be fixed. 
$\left(\mathrm{R}_{1}\right)$ For $\left(\lambda_{2}, \chi_{2}\right)$ in Regions $\mathrm{A}$ and $\mathrm{B}$ (including the boundaries but excluding the point $\left(\lambda_{1}, \chi_{1}\right)$ and the $\chi_{2}$-axis), there exist no positive steady states of (1.3). This is also true if $\chi_{2}$ is large enough (with respect to $\lambda_{2}$ ). See Theorems 3.1 and 3.3.

$\left(\mathrm{R}_{2}\right)$ For $\left(\lambda_{2}, \chi_{2}\right)$ in Region $\mathrm{A},(\underline{u}(x), 0, \underline{w}(x))$ is unstable, and if $(\underline{u}(x), \underline{w}(x))$ is locally asymptotically stable with respect to the single species dynamics (that is, (1.3) with $v \equiv 0)$, which is proved to be true for $\theta$ close to $k f(1)$, then for $\left(\lambda_{2}, \chi_{2}\right)$ in Region B, $(\underline{u}(x), 0, \underline{w}(x))$ is locally asymptotically stable. See Theorem 4.1.

$\left(R_{3}\right)$ There exists an increasing curve $\chi_{2}=\chi\left(\lambda_{2}\right), \lambda_{0} \leq \lambda_{2}<\infty$ as shown such that (i) for $\left(\lambda_{2}, \chi_{2}\right)$ above the curve, or $0<\lambda_{2}<\lambda_{0}$, or $\lambda_{2}=\lambda_{0}$ and $\chi_{2}>0$, $(\bar{u}(x), \bar{v}(x), 0)$ is unstable; (ii) if $\theta$ is less than but close to $k f(1)$, then for $\left(\lambda_{2}, \chi_{2}\right)$ below the curve, $(\bar{u}(x), \bar{v}(x), 0)$ is stable; moreover, for each fixed $\lambda_{2}>\lambda_{0}$, there exists a continuum $C$ of positive steady states $\left(\lambda_{2},(u, v, w)\right)$, joining two semitrivial steady states $\left(\left(\chi\left(\lambda_{2}\right),(\bar{u}, \bar{v}, 0)\right)\right.$ and $\left(\chi_{2}^{\infty},(\underline{u}, 0, \underline{w})\right)$. See Theorems 4.6 and 5.6.

$\left(\mathrm{R}_{4}\right)$ The positive steady states $\left(\chi_{2},(u, v, w)\right)$ near $\left(\chi\left(\lambda_{2}\right),(\bar{u}, \bar{v}, 0)\right)$ are locally asymptotically stable and satisfy $\chi\left(\lambda_{2}\right)<\chi_{2}$ if $\theta$ is close to $k f(1)$ and $\lambda_{2} \neq \lambda_{1}$. See Theorem 6.13 .

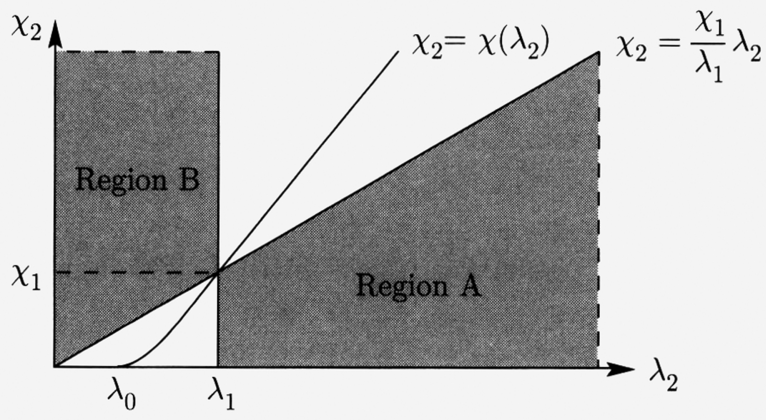

When the semitrivial or positive steady states are stable, we suspect that they are actually globally stable. These results indicate that (i) for $\left(\lambda_{2}, \chi_{2}\right)$ below the curve $\chi_{2}=\chi\left(\lambda_{2}\right)$, the coexistence of the competing species is impossible and the $v$-species survives and the $w$-species gets wiped out; (ii) the stable coexistence is possible if $\chi_{2}$ is larger (slightly, but not too much) than $\chi\left(\lambda_{2}\right)$; (iii) if $\chi_{2}$ is too large, the $w$-species prevails against the $v$-species by wiping it out.

Our study in (1.3) is motivated by [LC], where (i) both $\chi_{1}$ and $\chi_{2}$ are taken to be zero, (ii) the boundary condition of $u$ at $x=1$ is of Dirichlet type, (iii) and different consumption and birth rates, with $f(u)$ being a step function, are also assumed. Since $f(u)$ is assumed to be a step function, explicit formulas for the steady states are obtained. No stability analysis of steady states (trivial or nontrivial) was given.

To our knowledge, the global stability of steady states, due solely to the effect of diffusion, is established only in [DHMP] (for the Lotka-Voltera competition model with nonhomogeneous habitat). The story is that when the comparison principle applies (so the system is monotone), then it is possible to establish the global stability of semitrivial steady states; when the system is not monotone (such as in our case) or a small perturbation of such, the global stability still remains an open problem. 


\section{Global solutions in time and stability of the trivial steady state.}

Theorem 2.1 (Global Existence and Boundedness). For any $u_{0}, v_{0}$, and $w_{0}$ in $H^{1}(0,1)$ satisfying $1 \geq u_{0}>0, v_{0} \geq 0, \not \equiv 0, w_{0} \geq 0, \not \equiv 0$ on $[0,1],(1.3)$ with the initial condition $\left.(u, v, w)\right|_{t=0}=\left(u_{0}, v_{0}, w_{0}\right)$ has a unique positive global-in-time solution $(u, v, w)$ such that

(i) $(u(\cdot, t), v(\cdot, t), w(\cdot, t)) \in C\left([0, \infty), H^{1}(0,1) \times H^{1}(0,1) \times H^{1}(0,1)\right)$,

$$
(u, v, w) \in C_{\mathrm{loc}}^{2+2 \varepsilon, 1+\varepsilon}([0,1] \times(0, \infty))
$$

(ii) $0<u<1, v>0$ and $w>0$ are bounded on $[0,1] \times(0, \infty)$.

Theorem 2.2 (Stability of Trivial Steady State). (i) Suppose $k f(1) \leq \theta$. Then in the $L^{\infty}$-topology, $(u, v, w)=(1,0,0)$ attracts every positive solution of $(1.3)$ with the initial value satisfying the condition in Theorem 2.1. Moreover, if $k f(1)<\theta$, then

$$
\begin{gathered}
\|v(\cdot, t)\|_{\infty}+\|w(\cdot, t)\|_{\infty} \leq C \exp ((k f(1)-\theta) t), \quad t \geq 0, \\
\|1-u(\cdot, t)\|_{\infty} \leq C \exp (-\min (a, \theta-k f(1)) t), \quad t \geq 0,
\end{gathered}
$$

where $a$ is any number less than the first eigenvalue of $-d^{2} / d x^{2}$ with the boundary condition $u^{\prime}(0)=0=u^{\prime}(1)+h u(1)$.

(ii) Suppose $k f(1)>\theta$. Then $(u, v, w)=(1,0,0)$ is unstable in the $L^{\infty}$-topology.

These two theorems can be proved by slightly modifying the proofs for the single species case (see Theorems 4.8 and 5.1 in [W]).

3. The nonexistence of positive steady states. The nonnegative steady states of (1.3) satisfy

$$
\begin{cases}u^{\prime \prime}=f(u)(v+w), & x \in(0,1), \\ \left(\lambda_{1} v^{\prime}-\chi_{1} \phi^{\prime}(u) u^{\prime} v\right)^{\prime}+(k f(u)-\theta) v=0, & x \in(0,1), \\ \left(\lambda_{2} w^{\prime}-\chi_{2} \phi^{\prime}(u) u^{\prime} w\right)^{\prime}+(k f(u)-\theta) w=0, & x \in(0,1), \\ u^{\prime}(0)=0, \quad u^{\prime}(1)=h(1-u(1)), & \\ \lambda_{1} v^{\prime}-\chi_{1} \phi^{\prime}(u) u^{\prime} v=0=\lambda_{2} w^{\prime}-\chi_{2} \phi^{\prime}(u) u^{\prime} w, & x=0,1, \\ u \geq 0, \quad v \geq 0, \quad w \geq 0, & x \in[0,1] .\end{cases}
$$

Theorem 2.1 implies that the only solution of $(3.1)$ is the trivial one $(u, v, w)=(1,0,0)$ if $\theta \geq k f(1)$.

From now on, we assume $0<\theta<k f(1)$.

Theorem 3.1. Let $\lambda_{1}>0$ and $\chi_{1} \geq 0$ be fixed. For $\left(\lambda_{2}, \chi_{2}\right)$ in the Regions A and B (including the boundaries except the point $\left(\lambda_{1}, \chi_{1}\right)$ and $\chi_{2}$-axis), (1.3) has no positive steady states.

Proof. We argue by contradiction. Suppose $(u, v, w)$ is a positive solution of (3.1). Then $0<u<1$ on $[0,1], u^{\prime}>0, v^{\prime}>0, w^{\prime}>0$ on $(0,1)$.

Let

$$
z_{1}=v e^{-\chi_{1} \phi(u) / \lambda_{1}}, \quad \text { and } \quad z_{2}=w e^{-\chi_{2} \phi(u) / \lambda_{2}} .
$$


Then

$$
\begin{cases}\lambda_{1}\left(z_{1}^{\prime} e^{\chi_{1} \phi(u) / \lambda_{1}}\right)^{\prime}+(k f(u)-\theta) z_{1} e^{\chi_{1} \phi(u) / \lambda_{1}}=0, & x \in(0,1), \\ \lambda_{2}\left(z_{2}^{\prime} e^{\chi_{2} \phi(u) / \lambda_{2}}\right)^{\prime}+(k f(u)-\theta) z_{2} e^{\chi_{2} \phi(u) / \lambda_{2}}=0, & x \in(0,1), \\ z_{1}^{\prime}(0)=z_{1}^{\prime}(1)=0=z_{2}^{\prime}(0)=z_{2}^{\prime}(1) . & \end{cases}
$$

Since $\int_{0}^{1}(k f(u)-\theta) z_{1} e^{\chi_{1} \phi(u) / \lambda_{1}} d x=0$, and $f(u(x))$ is increasing, $z_{1}^{\prime}>0$ on $(0,1)$. Similarly, $z_{2}^{\prime}>0$ on $(0,1)$.

Multiplying the $z_{1}$-equation in (3.3) by $z_{2}$ and integrating by parts, we have

$$
\int_{0}^{1}\left[-\lambda_{1} e^{\chi_{1} \phi(u) / \lambda_{1}} z_{1}^{\prime} z_{2}^{\prime}+(k f(u)-\theta) e^{\chi_{1} \phi(u) / \lambda_{1}} z_{1} z_{2}\right] d x=0 .
$$

Multiplying the $z_{2}$-equation by $z_{1} e^{\left(\frac{\chi_{1}}{\lambda_{1}}-\frac{\chi_{2}}{\lambda_{2}}\right) \phi(u)}$, we have

$$
\int_{0}^{1}\left[-\lambda_{2} e^{\chi_{2} \phi(u) / \lambda_{2}} z_{2}^{\prime}\left(z_{1} e^{\left(\frac{\chi_{1}}{\lambda_{1}}-\frac{\chi_{2}}{\lambda_{2}}\right) \phi(u)}\right)^{\prime}+(k f(u)-\theta) e^{\chi_{1} \phi(u) / \lambda_{1}} z_{1} z_{2}\right] d x=0 .
$$

Subtracting (3.5) from (3.4), we obtain

$$
\left(\lambda_{2}-\lambda_{1}\right) \int_{0}^{1} z_{1}^{\prime} z_{2}^{\prime} e^{\chi_{1} \phi(u) / \lambda_{1}} d x+\lambda_{2}\left(\frac{\chi_{1}}{\lambda_{1}}-\frac{\chi_{2}}{\lambda_{2}}\right) \int_{0}^{1} z_{2}^{\prime} z_{1} \phi^{\prime}(u) u^{\prime} e^{\chi_{1} \phi(u) / \lambda_{1}} d x=0 .
$$

But for $\left(\lambda_{2}, \chi_{2}\right)$ in Regions $\mathrm{A}$ and $\mathrm{B}$, both terms in (3.6) either have the same sign, or one is zero while the other is not. This completes the proof of Theorem 3.1.

REMARK 3.2. If $\left(\lambda_{1}, \chi_{1}\right)=\left(\lambda_{2}, \chi_{2}\right)$, (3.1) has infinitely many positive solutions $(\bar{u}, \alpha \bar{v},(1-\alpha) \bar{v})$, where $\alpha$ is an arbitrary constant in $(0,1)$.

Theorem 3.3. For fixed $\lambda_{1}>0, \lambda_{2}>0$, and $\chi_{1} \geq 0,(1.3)$ has no positive steady states for $\chi_{2}$ large.

Proof. Suppose there exists a sequence of $\chi_{2} \rightarrow \infty$ such that (3.1) has a positive solution $(u, v, w)$. Since $0<u<1$ and $u^{\prime}>0$ is bounded on $[0,1]$, there exists a subsequence of $\chi_{2} \rightarrow \infty$ such that $u \rightarrow$ some $u_{\infty}$ in $C^{0}[0,1]$.

Adding the $v$-equation and $w$-equation in (3.1), we have

$$
\begin{aligned}
\theta \int_{0}^{1}(v+w) d x & =k \int_{0}^{1} f(u)(v+w) d x \\
& =k u^{\prime}(1)=k h(1-u(1))<k h .
\end{aligned}
$$

From this and the fact that $v^{\prime}>0, w^{\prime}>0$ in $(0,1)$, it follows that $v$ and $w$ are bounded on any $[0,1-\delta], \delta>0$ small. In fact, by Lemma 2.2 of $[\mathrm{W}], v$ is bounded on $[0,1]$ as $\chi_{2} \rightarrow \infty$. Thus $u^{\prime}(x)=\int_{0}^{x} f(u)(v+w) d x$ is equi-continuous on $[0,1-\delta]$ and hence

$$
u \rightarrow u_{\infty} \quad \text { in } C_{\mathrm{loc}}^{1}[0,1) \cap C^{0}[0,1]
$$

Since

$$
\lambda_{1} v^{\prime}(x)=\chi_{1} u^{\prime}(x) \phi^{\prime}(u(x)) v(x)+\int_{0}^{x}(k f(u)-\theta) v d y
$$

by (3.8), we have

$$
v \rightarrow \text { some } v_{\infty} \quad \text { in } C_{\mathrm{loc}}^{1}[0,1) \cap C^{0}[0,1]
$$


By the fact that $w$ is increasing and bounded on $[0,1-\delta]$ and by Helly's Theorem, we have that after passing to a subsequence,

$$
w \rightarrow \text { some } w_{\infty} \text { pointwise on }[0,1) .
$$

Now we see that $u_{\infty}$ and $v_{\infty}$ satisfy

$$
\begin{cases}u_{\infty}^{\prime}(x)=\int_{0}^{x} f\left(u_{\infty}\right)\left(v_{\infty}+w_{\infty}\right) d x, & x \in(0,1), \\ u_{\infty}^{\prime}(0)=0, \quad u_{\infty}^{\prime}(1)=h\left(1-u_{\infty}(1)\right), & \\ \left(\lambda_{1} v_{\infty}^{\prime}-\chi_{1} u_{\infty}^{\prime} \phi^{\prime}\left(u_{\infty}\right) v_{\infty}\right)^{\prime}+\left(k f\left(u_{\infty}\right)-\theta\right) v_{\infty}=0, & x \in(0,1), \\ \lambda_{1} v_{\infty}^{\prime}-\chi_{1} u_{\infty}^{\prime} \phi^{\prime}\left(u_{\infty}\right) v_{\infty}=0, & x=0,1 .\end{cases}
$$

Here $u_{\infty}^{\prime}(1)$ and $v_{\infty}^{\prime}(1)$ are defined by the left-hand limits at $x=1$. Integrating the $w$-equation in (3.1) twice, we have

$$
\begin{aligned}
\lambda_{2}(w(x)-w(0))-\chi_{2} \int_{0}^{x} u^{\prime}(x) \phi^{\prime}(u(x)) & w(x) d x \\
& +\int_{0}^{x} \int_{0}^{y}(k f(u(\tau))-\theta) w(\tau) d \tau d y=0 .
\end{aligned}
$$

Dividing this by $\chi_{2}$ and then sending it to infinity, we see that

$$
\int_{0}^{x} u_{\infty}^{\prime}(y) \phi^{\prime}\left(u_{\infty}(y)\right) w_{\infty}(y) d y=0
$$

and hence

$$
u_{\infty}^{\prime}(x) \phi^{\prime}\left(u_{\infty}(x)\right) w_{\infty}(x)=0, \quad \text { for } x \in(0,1)
$$

Note that $u_{\infty}, v_{\infty}$, and $w_{\infty}$ are nondecreasing on $[0,1]$. We claim that $w_{\infty} \equiv 0$ on $[0,1)$. Otherwise, there exists $x_{0} \in[0,1)$ such that $w_{\infty}>0$ on $\left(x_{0}, 1\right)$. Then (3.14) implies that $u_{\infty}^{\prime} \equiv 0$ on $\left(x_{0}, 1\right)$ and hence on $[0,1)$. On the other hand, $u_{\infty}^{\prime}(1)=h\left(1-u_{\infty}(1)\right)$. So $u_{\infty} \equiv 1$ on $[0,1]$. This contradicts (3.8) and the assumption $\theta<k f(1)$ and the fact

$$
\int_{0}^{1}(k f(u)-\theta) v d x=0 .
$$

We now claim that $v_{\infty}>0$ on $[0,1]$. If not, by the strong maximum principle and the Hopf boundary point lemma, we see that $v_{\infty} \equiv 0$ on $[0,1]$. Therefore, $u_{\infty} \equiv 1$ on $[0,1]$, which is again impossible.

Using the notation in the proof of Theorem 3.1, we are going to show a contradiction to (3.6) for $\chi_{2}$ large. By (3.3), we have

$$
\begin{aligned}
0<\lambda_{1} e^{\chi_{1} \phi(u(x)) / \lambda_{1}} z_{1}^{\prime}(x) & =-\int_{0}^{x}(k f(u)-\theta) z_{1} e^{\chi_{1} \phi(u) / \lambda_{1}} d y \\
& \leq \theta x z_{1}(x) e^{\chi_{1} \phi(u(x)) / \lambda_{1}},
\end{aligned}
$$

and hence

$$
0<z_{1}^{\prime}(x) \leq \frac{\theta}{\lambda_{1}} x z_{1}(x), \quad x \in(0,1)
$$


Since $u_{\infty}>0$ and $v_{\infty}>0$ on $[0,1]$, we see that

$$
u^{\prime}(x)=\int_{0}^{x} f(u)(v+w) d y \geq \int_{0}^{x} f(u) v d y \geq C_{1} x, \quad x \in(0,1),
$$

where $C_{1}$ is a positive constant independent of $\chi_{2}$.

Inequalities (3.16) and (3.17) contradict (3.6) for $\chi_{2}$ large. This completes the proof of Theorem 3.3.

4. Stability and instability of semitrivial steady states. As mentioned before, by the existence result of $[\mathrm{Z}]$ in the single species case, (1.3) has semitrivial steady states $(\bar{u}, \bar{v}, 0)$ and $(\underline{u}, 0, \underline{w})$. If $\theta$ is close to $k f(1)$, by $[\mathrm{W}],(\bar{u}, \bar{v})$ is unique and is locally exponentially asymptotically stable in the $H^{1}(0,1)$-topology with respect to the single species dynamics (that is, with respect to (1.3) with $w \equiv 0)$. The same is true for $(\underline{u}, \underline{w})$. For $\theta$ not close to $k f(1)$, the uniqueness and the stability of $(\bar{u}, \bar{v})$ and $(\underline{u}, \underline{w})$ are not known.

In the sequel, we use $(\bar{u}, \bar{v}, 0)$ (and $(\underline{u}, 0, \underline{w}))$ to denote any semitrivial steady state of (1.3).

Theorem 4.1. For $\left(\lambda_{2}, \chi_{2}\right)$ in Region A (including the boundary except point $\left(\lambda_{1}, \chi_{1}\right)$ ), the semitrivial steady state $(\underline{u}, 0, \underline{w})$ is unstable in the $H^{1}(0,1)$-topology, and if $(\bar{u}, \bar{v})$ is locally exponentially asymptotically stable with respect to the single species dynamics, then so is $(\bar{u}, \bar{v}, 0)$. The same is true in Region B if we exchange $(\underline{u}, 0, \underline{w})$ and $(\bar{u}, \bar{v}, 0)$.

Proof. Linearize $(3.1)$ at $(\underline{u}, 0, \underline{w})$. By the principle of linearized stability [S, Theorem 5.3], to show the instability of $(\underline{u}, 0, \underline{w})$, we only need to show the existence of an eigenvalue, with positive real part, of the following eigenvalue problem:

$$
\begin{cases}u^{\prime \prime}-f^{\prime}(\underline{u}) u \underline{w}-f(\underline{u})(v+w)=\eta u, & x \in(0,1), \\ u^{\prime}(0)=0=u^{\prime}(1)+h u(1), & \\ \left(\lambda_{1} v^{\prime}-\chi_{1} \phi^{\prime}(\underline{u}) \underline{u}^{\prime} v\right)^{\prime}+(k f(\underline{u})-\theta) v=\eta v, & x \in(0,1), \\ \lambda_{1} v^{\prime}-\chi_{1} \phi^{\prime}(\underline{u}) \underline{u}^{\prime} v=0, & x=0,1, \\ \left(\lambda_{2} w^{\prime}-\chi_{2} \phi^{\prime}(\underline{u}) \underline{u}^{\prime} w-\chi_{2} \phi^{\prime}(\underline{u}) u^{\prime} \underline{w}-\chi_{2} \phi^{\prime \prime}(\underline{u}) \underline{u^{\prime}} \underline{w} u\right)^{\prime} & \\ \quad+(k f(\underline{u})-\theta) w+k f^{\prime}(\underline{u}) u \underline{w}=\eta w, & x \in(0,1), \\ \lambda_{2} w^{\prime}-\chi_{2} \phi^{\prime}(\underline{u}) \underline{u}^{\prime} w-\chi_{2} \phi^{\prime}(\underline{u}) u^{\prime} \underline{w}-\chi_{2} \phi^{\prime \prime}(\underline{u}) \underline{u^{\prime}} \underline{w} u=0, & x=0,1 .\end{cases}
$$

If (4.1) with $v \equiv 0$ has an eigenvalue with positive real part, then we are done. So we assume that all the eigenvalues of (4.1) with $v \equiv 0$ have real parts no bigger than 0 .

Consider the $v$-eigenvalue problem embedded in (4.1).

Let $z=v e^{-\chi_{1} \phi(\underline{u}) / \lambda_{1}}$. Then this eigenvalue problem is equivalent to the following:

$$
\left\{\begin{array}{l}
\lambda_{1}\left(z^{\prime} e^{\chi_{1} \phi(\underline{u}) / \lambda_{1}}\right)^{\prime}+(k f(\underline{u})-\theta) z e^{\chi_{1} \phi(\underline{u}) / \lambda_{1}}=\eta e^{\chi_{1} \phi(\underline{u}) / \lambda_{1}} z, \quad x \in(0,1), \\
z^{\prime}(0)=0=z^{\prime}(1) .
\end{array}\right.
$$


The eigenvalues are real and can be characterized by the standard minmax procedure. In particular, the largest eigenvalue is given by

$$
\eta_{1}=-\inf _{\substack{z \in H^{1}(0,1) \\ z \neq \equiv 0}} \frac{\int_{0}^{1}\left(\lambda_{1}\left(z^{\prime}\right)^{2}-(k f(\underline{u})-\theta) z^{2}\right) e^{\chi_{1} \phi(\underline{u}) / \lambda_{1}} d x}{\int_{0}^{1} z^{2} e^{\chi_{1} \phi(\underline{u}) / \lambda_{1}} d x},
$$

and the associated eigenfunction $z_{1}$ must be of one sign, which we take to be positive. As before, it is easy to see that $z_{1}^{\prime}>0$ in $(0,1)$.

If $\eta_{1} \leq 0$, we reach a contradiction as follows. In (3.2), replace $w$ by $\underline{w}$. Then we have (3.6) with "=" replaced by " $\leq$ ", which is impossible for $\left(\lambda_{2}, \chi_{2}\right)$ in Region A, including the boundary, except point $\left(\lambda_{1}, \chi_{1}\right)$. Thus $\eta_{1}>0$.

Now let $v_{1}=z_{1} e^{\chi_{1} \phi(\underline{u}) / \lambda_{1}}$. Since the real part of all the eigenvalues of (4.1) with $v \equiv 0$ is assumed to be nompositive, by the Fredholm alternatives, (4.1) with $\eta=\eta_{1}$ and $v=v_{1}$ has a unique solution $\left(u_{1}, v_{1}, w_{1}\right)$. This means $\eta_{1}>0$ is an eigenvalue of (4.1) and hence $(\underline{u}, 0, \underline{w})$ is unstable.

We now proceed to show the stability of $(\bar{u}, \bar{v}, 0)$ for $\left(\lambda_{2}, \chi_{2}\right)$ in Region A. Linearize (3.1) at $(\bar{u}, \bar{v}, 0)$ to obtain the following eigenvalue problem:

$$
\begin{cases}u^{\prime \prime}-f^{\prime}(\bar{u}) u \bar{v}-f(\bar{u})(v+w)=\eta u, & x \in(0,1), \\ u^{\prime}(0)=0=u^{\prime}(1)+h u(1), & \\ \left(\lambda_{1} v^{\prime}-\chi_{1} \bar{u}^{\prime} \phi^{\prime}(\bar{u}) v-\chi_{1} \phi^{\prime}(\bar{u}) u^{\prime} \bar{v}-\chi_{1} \phi^{\prime \prime}(\bar{u}) \bar{u}^{\prime} \bar{v} u\right)^{\prime} & \\ \quad+k f^{\prime}(\bar{u}) \bar{v} u+(k f(\bar{u})-\theta) v=\eta v, & x \in(0,1), \\ \left(\lambda_{2} w^{\prime}-\chi_{2} \bar{u}^{\prime} \phi^{\prime}(\bar{u}) w\right)^{\prime}+(k f(\bar{u})-\theta) w=\eta w, & x \in(0,1), \\ \lambda_{1} v^{\prime}-\chi_{1} \bar{u}^{\prime} \phi^{\prime}(\bar{u}) v-\chi_{1} \phi^{\prime}(\bar{u}) u^{\prime} \bar{v}-\chi_{1} \phi^{\prime \prime}(\bar{u}) \bar{u}^{\prime} \bar{v} u=0, & x=0,1, \\ \lambda_{2} w^{\prime}-\chi_{2} \bar{u}^{\prime} \phi^{\prime}(\bar{u}) w=0, & x=0,1 .\end{cases}
$$

Since $(\bar{u}, \bar{v})$ is assumed to be exponentially stable with respect to single species dynamics, the real part of all eigenvalues of (4.3) with $w=0$ is negative. We need only to show that the largest eigenvalue of the $w$-eigenvalue problem in (4.3) is negative. Denote this eigenvalue by $\eta^{*}$ and let $z=w e^{-\chi_{2} \phi(\bar{u}) / \lambda_{2}}$. Then $z$ satisfies

$$
\left\{\begin{array}{l}
\lambda_{2}\left(z^{\prime} e^{\chi_{2} \phi(\bar{u}) / \lambda_{2}}\right)^{\prime}+(k f(\bar{u})-\theta) z e^{\chi_{2} \phi(\bar{u}) / \lambda_{2}}=\eta^{*} z e^{\chi_{2} \phi(\bar{u}) / \lambda_{2}}, \quad x \in(0,1), \\
z^{\prime}(0)=0=z^{\prime}(1) .
\end{array}\right.
$$

As in the case of $\eta_{1}$, we can show $\eta^{*}<0$ for $\left(\lambda_{2}, \chi_{2}\right)$ in Region A, including the boundary except the point $\left(\lambda_{1}, \chi_{1}\right)$. Theorem 4.1 is proved.

We shall show that there exists a curve that divides the first quadrant of the $\left(\lambda_{2}, \chi_{2}\right)$ plane into two parts such that for $\left(\lambda_{2}, \chi_{2}\right)$ in one part, $(\bar{u}, \bar{v}, 0)$ is stable; while in the other, it is unstable. To this end, we need to study the dependence of $\eta^{*}$ on $\left(\lambda_{2}, \chi_{2}\right) . \eta^{*}$ is given by

$$
\eta^{*}=-\inf _{\substack{z \in H^{1}(0,1) \\ z \neq 0}} \frac{\int_{0}^{1}\left(\lambda_{2}\left(z^{\prime}\right)^{2}-(k f(\bar{u})-\theta) z^{2}\right) e^{\chi_{2} \phi(\bar{u}) / \lambda_{2}} d x}{\int_{0}^{1} z^{2} e^{\chi_{2} \phi(\bar{u}) / \lambda_{2}} d x} .
$$

LEMma 4.2. $\eta^{*}$ is a continuous function of $\left(\lambda_{2}, \chi_{2}\right) \in(0, \infty) \times[0, \infty)$; it is increasing in $\chi_{2}$ and decreasing in $\lambda_{2}$. 
Proof. The continuity of $\eta^{*}$ is easy to prove by using a standard argument. Let $\eta^{(1)}=\eta^{*}\left(\lambda_{2}^{(1)}, \chi_{2}^{(1)}\right)$ and $\eta^{(2)}=\eta^{*}\left(\lambda_{2}^{(2)}, \chi_{2}^{(2)}\right)$.

Let $z_{1}$ and $z_{2}$ be the positive eigenfunctions of (4.4) corresponding to $\eta^{(1)}$ and $\eta^{(2)}$, respectively. Then $z_{1}^{\prime}>0$ and $z_{2}^{\prime}>0$ on $(0,1)$ and the following analog of $(3.6)$ holds:

$$
\begin{aligned}
\left(\lambda_{2}^{(2)}-\right. & \left.\lambda_{2}^{(1)}\right) \int_{0}^{1} z_{1}^{\prime} z_{2}^{\prime} e^{\chi_{2}^{(1)} \phi(\bar{u}) / \lambda_{2}^{(1)}} d x+\lambda_{2}^{(2)}\left(\frac{\chi_{2}^{(1)}}{\lambda_{2}^{(1)}}-\frac{\chi_{2}^{(2)}}{\lambda_{2}^{(2)}}\right) \\
& \times \int_{0}^{1} z_{2}^{\prime} z_{1} \bar{u}^{\prime} \phi^{\prime}(\bar{u}) e^{\chi_{2}^{(1)} \phi(\bar{u}) / \lambda_{2}^{(1)}} d x=\left(\eta^{(1)}-\eta^{(2)}\right) \int_{0}^{1} z_{1} z_{2} e^{\chi_{2}^{(1)} \phi(\bar{u}) / \lambda_{2}^{(1)}} d x .
\end{aligned}
$$

From this, the desired monotonicity of $\eta^{*}$ follows.

Lemma 4.3. For fixed $\lambda_{1}, \lambda_{2}>0$ and $\chi_{1} \geq 0, \eta^{*}>0$ if $\chi_{2}$ is large.

Proof. Let $\bar{V}=\bar{v} e^{-\chi_{1} \phi(\bar{u}) / \lambda_{1}}$ and $z_{2}$ be a positive eigenfunction of (4.4) with $\eta=\eta^{*}$. Then we have the following analog of (3.6):

$$
\begin{aligned}
\eta^{*} \int_{0}^{1} \bar{V} z_{2} e^{\chi_{1} \phi(\bar{u}) / \lambda_{1}} d x=\lambda_{2}\left(\frac{\chi_{2}}{\lambda_{2}}-\frac{\chi_{1}}{\lambda_{1}}\right) \int_{0}^{1} z_{2}^{\prime} \bar{V} \bar{u}^{\prime} \phi^{\prime}(\bar{u}) e^{\chi_{1} \phi(\bar{u}) / \lambda_{1}} d x \\
\\
+\left(\lambda_{1}-\lambda_{2}\right) \int_{0}^{1} \bar{V}^{\prime} z_{2}^{\prime} e^{\chi_{1} \phi(\bar{u}) / \lambda_{1}} d x .
\end{aligned}
$$

On the other hand, we have the analogs of (3.16) and (3.17), which combined with (4.7), lead to $\eta^{*}>0$ for $\chi_{2}$ large. The proof of Lemma 4.3 is complete.

By the proof of Theorem 4.1, $\eta^{*}\left(\lambda_{2}, \chi_{2}\right)<0$ for $\left(\lambda_{2}, \chi_{2}\right)$ in Region A, including the boundary, except the point $\left(\lambda_{1}, \chi_{1}\right)$; in particular, if $\chi_{1}>0, \eta^{*}\left(\lambda_{2}, 0\right)<0$ for $\lambda_{2} \geq \lambda_{1}$, and if $\chi_{1}=0, \eta^{*}\left(\lambda_{2}, 0\right)<0$ for $\lambda_{2}>\lambda_{1}$ and $\eta^{*}\left(\lambda_{1}, 0\right)=0$.

Define

$$
\lambda_{0}=\inf \left\{\lambda_{2}>0 \mid \eta^{*}\left(\lambda_{2}, 0\right)<0\right\}
$$

Lemma 4.4. $\lambda_{0}>0$.

Proof. Suppose $\chi_{1}=0$. Then $\lambda_{0}=\lambda_{1}$ because $\eta^{*}\left(\lambda_{1}, 0\right)=0$ and $\eta^{*}$ is decreasing with respect to $\lambda_{2}$.

Now suppose $\chi_{1}>0$. Observe that ( $\bar{V}$ as given in the proof of Lemma 4.3)

$$
\begin{aligned}
\eta^{*}\left(\lambda_{2}, 0\right) & =-\inf _{\substack{z \in H^{1}(0,1) \\
z \neq 0}} \frac{\int_{0}^{1}\left(\lambda_{2}\left(z^{\prime}\right)^{2}-(k f(\bar{u})-\theta) z^{2}\right) d x}{\int_{0}^{1} z^{2} d x}, \\
& \geq \frac{-\int_{0}^{1} \lambda_{2}\left[\left(\bar{V} e^{\chi_{1} \phi(\bar{u}) / 2 \lambda_{1}}\right)^{\prime}\right]^{2} d x+\int_{0}^{1}(k f(\bar{u})-\theta) \bar{V}^{2} e^{\chi_{1} \phi(\bar{u}) / \lambda_{1}} d x}{\int_{0}^{1}\left(\bar{V} e^{\chi_{1} \phi(\bar{u}) / 2 \lambda_{1}}\right)^{2} d x} .
\end{aligned}
$$

The second integral in the numerator is equal to $\int_{0}^{1} \lambda_{1}\left(\bar{V}^{\prime}\right)^{2} e^{\chi_{1} \phi(\bar{u}) / \lambda_{1}} d x$ (see (3.3)). Thus if $\lambda_{2}$ is small, $\eta^{*}\left(\lambda_{2}, 0\right)>0, \lambda_{0}>0$. The proof of Lemma 4.4 is complete.

LEMmA 4.5. There exists a continuous increasing function $\chi_{2}=\chi\left(\lambda_{2}\right), \lambda_{0}<\lambda_{2}<\infty$ such that (i) $\eta^{*}\left(\lambda_{2}, \chi\left(\lambda_{2}\right)\right)=0$, (ii) the graph of $\chi_{2}=\chi\left(\lambda_{2}\right)$ is strictly above Region A if $\lambda_{2}>\lambda_{1}$ and strictly below Region $\mathrm{B}$ for $\lambda_{0}<\lambda_{2}<\lambda_{1}$. 
Proof. By Lemmas 4.2-4.4, for any $\lambda_{2}>\lambda_{0}$, there exists a unique $\chi\left(\lambda_{2}\right)$ such that $\eta^{*}\left(\lambda_{2}, \chi\left(\lambda_{2}\right)\right)=0$. By the continuity of $\eta^{*}$ on $\left(\lambda_{2}, \chi_{2}\right), \chi\left(\lambda_{2}\right)$ is a continuous function of $\lambda_{2}>\lambda_{0}$. Since $\eta^{*}\left(\lambda_{2}, \chi_{2}\right)$ is negative for $\left(\lambda_{2}, \chi_{2}\right)$ in Region A (including the boundary except $\left.\left(\lambda_{1}, \chi_{1}\right)\right)$ and positive for $\left(\lambda_{2}, \chi_{2}\right)$ in Region B, we have (ii). The proof of Lemma 4.5 is complete.

ThEOREM 4.6. For $\left(\lambda_{2}, \chi_{2}\right)$ above the graph of $\chi_{2}=\chi\left(\lambda_{2}\right)$, or $0<\lambda_{2}<\lambda_{0}$, or $\lambda_{2}=\lambda_{0}$ and $\chi_{2}>0,(\bar{u}, \bar{v}, 0)$ is unstable in $H^{1}(0,1)$. For $\left(\lambda_{2}, \chi_{2}\right)$ below the graph, $(\bar{u}, \bar{v}, 0)$ is stable in $H^{1}(0,1)$, provided $(\bar{u}, \bar{v})$ is stable with respect to the single species dynamics (i.e., (1.3) with $w \equiv 0$ ).

Proof. As in the proof of Theorem 4.1, to show the instability and stability, all we need to show are (i) $\eta^{*}\left(\lambda_{2}, \chi_{2}\right)>0$ for $\left(\lambda_{2}, \chi_{2}\right)$ above the graph of $\chi_{2}=\chi\left(\lambda_{2}\right)$, or $0<\lambda_{2}<\lambda_{0}$, or $\lambda_{2}=\lambda_{0}$ and $\chi_{2}>0$; (ii) $\eta^{*}\left(\lambda_{2}, \chi_{2}\right)<0$ for $\left(\lambda_{2}, \chi_{2}\right)$ below the graph of $\chi_{2}=\chi\left(\lambda_{2}\right)$. These follow from the definitions of $\chi\left(\lambda_{2}\right)$ and $\lambda_{0}$ and from Lemma 4.2.

5. Bifurcation of positive steady states. In this section, we prove the existence of positive solutions of (3.1) that bifurcate from the semitrivial solution $(\bar{u}, \bar{v}, 0) ; \chi_{2}$ will be the bifurcation parameter. We shall show first that local bifurcation occurs at $\chi_{2}=\chi\left(\lambda_{2}\right)$ for each fixed $\lambda_{2}>\lambda_{0}$. We substitute $u$ and $v$ in (3.1) by $u+\bar{u}$ and $v+\bar{v}$, respectively. The resulting system can be written as

$$
\begin{cases}u^{\prime \prime}=F^{0}(u, v, w)-\bar{u}^{\prime \prime}, & x \in(0,1), \\ \left(A^{1}(u, v)\right)^{\prime}+F^{1}(u, v)=0, & x \in(0,1), \\ \left(A^{2}(u, w)\right)^{\prime}+F^{2}(u, w)=0, & x \in(0,1), \\ u^{\prime}(0)=0=u^{\prime}(1)+h u(1), & \\ A^{1}(u, v)=0=A^{2}(u, w), & x=0,1,\end{cases}
$$

where $F^{0}(u, v, w)=f(u+\bar{u})(\bar{v}+v+w), F^{1}(u, v)=(k f(u+\bar{u})-\theta)(\bar{v}+v), F^{2}(u, w)=$ $(k f(u+\bar{u})-\theta) w, A^{1}(u, v)=\lambda_{1}(v+\bar{v})^{\prime}-\chi_{1}(\bar{u}+u)^{\prime} \phi^{\prime}(u+\bar{u})(v+\bar{v})$ and $A^{2}(u, w)=$ $\lambda_{2} w^{\prime}-\chi_{2}(\bar{u}+u)^{\prime} \phi^{\prime}(u+\bar{u}) w$.

For the time being, we extend $f$ and $\phi$ so that they have the same regularity over $(-\infty, \infty)$ as mentioned in (1.2). We now convert (5.1) to "integral" equations.

Note $\bar{u}^{\prime \prime}=F^{0}(0,0,0)$ and $F^{0}(u, v, w)=F^{0}(0,0,0)+\nabla F^{0}(0,0,0) \cdot(u, v, w)+R_{1}(u, v, w)$, where $R_{1}$ is a "higher-order term". Let $K_{1}$ be the inverse of $-d^{2} / d x^{2}$ with the $u$-boundary condition in (5.1).

Let $\mathrm{X}=C^{1+\alpha}[0,1]$. Then $K_{1}: C^{\alpha}[0,1] \rightarrow \mathrm{X}$ is linear and compact. The $u$-equation in $(5.1)$ is equivalent to

$$
u+K_{1}\left[f(\bar{u})(v+w)+f^{\prime}(\bar{u}) \bar{v} u\right]+K_{1} R_{1}(u, v, w)=0
$$

where $(u, v, w) \in \mathrm{X}^{3}=\mathrm{X} \times \mathrm{X} \times \mathrm{X}$ and $K_{1} R_{1}: \mathrm{X}^{3} \rightarrow \mathrm{X}$ is $C^{2}$ smooth and compact with

$$
\left\|K_{1} R_{1}(u, v, w)\right\|_{\mathrm{X}}=O\left(\|(u, v, w)\|_{\mathrm{X}^{3}}^{2}\right), \quad \text { as }\|(u, v, w)\|_{\mathrm{X}^{3}} \rightarrow 0 .
$$


Now we convert the $v$-equation in (5.1). Linearize both $A^{1}$ and $F^{1}$ at $(u, v)=(0,0)$ :

$$
\begin{gathered}
A^{1}(u, v)=A^{1}(0,0)+\left[A_{(u, v)}^{1}(0,0)\right](u, v)+S^{1}(u, v) \\
=A^{1}(0,0)+\left(\lambda_{1} v^{\prime}-\chi_{1} \bar{u}^{\prime} \phi^{\prime}(\bar{u}) v-\chi_{1} \phi^{\prime}(\bar{u}) u^{\prime} \bar{v}-\chi_{1} \phi^{\prime \prime}(\bar{u}) \bar{u}^{\prime} \bar{v} u\right)+S^{1}(u, v), \\
F^{1}(u, v)=F^{1}(0,0)+\nabla F^{1}(0,0) \cdot(u, v)+T^{1}(u, v) .
\end{gathered}
$$

When we differentiate $A^{1}(u, v)$ with respect to $x$, we have some terms with $u^{\prime \prime}$ as their factors. In such a scenario, we use the $u$-equation in (5.1) to replace $u^{\prime \prime}$ by $F^{0}(u, v, w)-$ $F^{0}(0,0,0)$. Now the $v$-equation in $(5.1)$ can be rewritten as

$$
\begin{aligned}
\left(\lambda_{1} v^{\prime}-\chi_{1} \bar{u}^{\prime} \phi^{\prime}(\bar{u}) v\right)^{\prime} & -\chi_{1}\left[\left(\bar{u}^{\prime} \bar{v} \phi^{\prime \prime}(\bar{u}) u\right)^{\prime}+\left(\bar{v} \phi^{\prime}(\bar{u})\right)^{\prime} u^{\prime}+\bar{v} \phi^{\prime}(\bar{u})(f(\bar{u})(v+w)\right. \\
& \left.\left.+f^{\prime}(\bar{u}) \bar{v} u\right)\right]+\left[k f^{\prime}(\bar{u}) u \bar{v}+(k f(\bar{u})-\theta) v\right]-R_{2}(u, v, w)=0
\end{aligned}
$$

where

$$
\left\|R_{2}(u, v, w)\right\|_{C^{\alpha}[0,1]}=O\left(\|(u, v, w)\|_{\mathrm{X}^{3}}^{2}\right) \quad \text { as }\|(u, v, w)\|_{\mathrm{X}^{3}} \rightarrow 0 .
$$

For any $g \in \mathrm{X}$, let $K_{2} g$ be the unique solution of

$$
\begin{cases}\left(-\lambda_{1} v^{\prime}+\chi_{1} \bar{u}^{\prime} \phi^{\prime}(\bar{u}) v\right)^{\prime}+v=g, & x \in(0,1), \\ -\lambda_{1} v^{\prime}+\chi_{1} \bar{u}^{\prime} \phi^{\prime}(\bar{u}) v=0, & x=0,1 .\end{cases}
$$

Then $K_{2}: C^{\alpha}[0,1] \rightarrow \mathrm{X}$ is linear and compact. For any $(u, v) \in \mathrm{X} \times \mathrm{X}$, let $B_{1}(u)$ be the unique solution of

$$
\begin{cases}\left(-\lambda_{1} z^{\prime}+\chi_{1} \bar{u}^{\prime} \phi^{\prime}(\bar{u}) z\right)^{\prime}+z=0, & x \in(0,1), \\ -\lambda_{1} z^{\prime}+\chi_{1} \bar{u}^{\prime} \phi^{\prime}(\bar{u}) z=\chi_{1} \phi^{\prime}(\bar{u}) \bar{v} u^{\prime}+\chi_{1} \phi^{\prime \prime}(\bar{u}) \bar{u}^{\prime} \bar{v} u, & x=0,1\end{cases}
$$

let $B_{2}(u, v)$ be the unique solution of

$$
\begin{cases}\left(-\lambda_{1} z^{\prime}+\chi_{1} \bar{u}^{\prime} \phi^{\prime}(\bar{u}) z\right)^{\prime}+z=0, & x \in(0,1), \\ -\lambda_{1} z^{\prime}+\chi_{1} \bar{u}^{\prime} \phi^{\prime}(\bar{u}) z=-S^{1}(u, v), & x=0,1 .\end{cases}
$$

Then $B_{1}: \mathrm{X} \rightarrow \mathrm{X}$ is linear and compact, and $B_{2}: \mathrm{X} \times \mathrm{X} \rightarrow \mathrm{X}$ is $C^{2}$ smooth and compact and $\left\|B_{2}(u, v)\right\|_{\mathrm{X}}=O\left(\|(u, v)\|_{\mathrm{X}_{2}}^{2}\right)$ as $\|(u, v)\|_{\mathrm{X}_{2}} \rightarrow 0$. Now the $v$-equation and the $v$-boundary condition in (5.1) can be written as

$$
\begin{array}{r}
v-K_{2} v+\chi_{1} K_{2}\left[\left(\bar{v} \phi^{\prime}(\bar{u})\right)^{\prime} u^{\prime}+\left(\bar{u}^{\prime} \bar{v} \phi^{\prime \prime}(\bar{u}) u\right)^{\prime}+\bar{v} \phi^{\prime}(\bar{u})\left(f(\bar{u})(v+w)+f^{\prime}(\bar{u}) \bar{v} u\right)\right] \\
-K_{2}\left[k f^{\prime}(\bar{u}) \bar{v} u+(k f(\bar{u})-\theta) v\right]+K_{2} R_{2}(u, v, w)+B_{1}(u)+B_{2}(u, v)=0
\end{array}
$$

where $(u, v, w) \in \mathrm{X}^{3}$ and the only nonlinear terms in (5.7) are $B_{2}(u, v)$ and $K_{2} R_{2}(u, v, w)$.

We now convert the $w$-equation in (5.1). Observe

$$
\begin{gathered}
A^{2}(u, w)=\lambda_{2} w^{\prime}-\chi_{2} \bar{u}^{\prime} \phi^{\prime}(\bar{u}) w-\chi_{2}\left[(\bar{u}+u)^{\prime} \phi^{\prime}(\bar{u}+u)-\bar{u}^{\prime} \phi^{\prime}(\bar{u})\right] w \\
=\lambda_{2} w^{\prime}-\chi_{2} \bar{u}^{\prime} \phi^{\prime}(\bar{u}) w-\chi_{2} S^{2}(u, w), \\
F^{2}(u, w)=(k f(\bar{u})-\theta) w+T^{2}(u, w),
\end{gathered}
$$


where $S^{2}$ and $T^{2}$ are higher-order terms. Now the $w$-equation can be written as

$$
\begin{aligned}
\left(\lambda_{2} w^{\prime}-\chi\left(\lambda_{2}\right) \bar{u}^{\prime} \phi^{\prime}(\bar{u}) w\right)^{\prime}+\left(\chi\left(\lambda_{2}\right)-\right. & \left.\chi_{2}\right)\left(\bar{u}^{\prime} \phi^{\prime}(\bar{u}) w\right)^{\prime} \\
& +(k f(\bar{u})-\theta) w+T^{2}(u, w)-\chi_{2}\left(S^{2}(u, w)\right)^{\prime}=0 .
\end{aligned}
$$

Again, when differentiating $S^{2}$ with respect to $x$, we have some terms with $u^{\prime \prime}$ as their factors, which we replace by $F^{0}(u, v, w)-F^{0}(0,0,0)$. Then

$$
\left\|\left(S^{2}(u, w)\right)^{\prime}\right\|_{C^{\alpha}[0,1]}=O\left(\|(u, w)\|_{\mathrm{X}^{2}}^{2}\right), \quad \text { as }\|(u, w)\|_{\mathrm{X}^{2}} \rightarrow 0 .
$$

Obviously, $\left\|T^{2}(u, w)\right\|_{C^{\alpha}[0,1]}=O\left(\|(u, w)\|_{\mathrm{X}^{2}}^{2}\right)$.

Define $K_{3} g$ by replacing in (5.4) $\lambda_{1}$ and $\chi_{1}$ by $\lambda_{2}$ and $\chi\left(\lambda_{2}\right)$, respectively; define $B_{3}(w)$ by replacing in (5.5) $\lambda_{1}$ by $\lambda_{2}, \chi_{1}$ by $\chi\left(\lambda_{2}\right)$, and the right-hand side of the boundary condition by $\bar{u}^{\prime} \phi^{\prime}(\bar{u}) w$; define $B_{4}(u, w)$ by replacing $\lambda_{1}, \chi_{1}$, and $-S^{1}$ by $\lambda_{2}, \chi\left(\lambda_{2}\right)$ and $S^{2}(u, w)$, respectively. Then $K_{3}: C^{\alpha}[0,1] \rightarrow \mathrm{X}$ and $B_{3}: C^{\alpha}[0,1] \rightarrow \mathrm{X}$ are linear and compact; $B_{4}: \mathrm{X} \times \mathrm{X} \rightarrow \mathrm{X}$ is $C^{2}$ smooth and compact, with $\left\|B_{4}(u, w)\right\|_{\mathrm{X}}=O\left(\|(u, w)\|_{\mathrm{X}^{2}}^{2}\right)$.

Now the $w$-equation can be converted to

$$
\begin{aligned}
w-K_{3}[(k f(\bar{u})-\theta) w+w]+\left(\chi_{2}-\chi\left(\lambda_{2}\right)\right) K_{3}\left[\left(\bar{u}^{\prime} \phi^{\prime}(\bar{u}) w\right)^{\prime}\right]-K_{3} T^{2}(u, w) \\
+\chi_{2} K_{3}\left[\left(S^{2}(u, w)\right)^{\prime}\right]+\left(\chi_{2}-\chi\left(\lambda_{2}\right)\right) B_{3}(w)+\chi_{2} B_{4}(u, w)=0,
\end{aligned}
$$

where $(u, v, w) \in \mathrm{X}^{3}$.

Let $F\left(\chi_{2},(u, v, w)\right)$ be the vector in $\mathrm{X}^{3}$, defined by the left-hand sides of $(5.2),(5.3)$, and (5.8). Then (5.1) is equivalent to

$$
F\left(\chi_{2},(u, v, w)\right)=0, \quad(u, v, w) \in \mathrm{X}^{3} .
$$

Observe $F\left(\chi_{2},(0,0,0)\right)=0$ and, by the regularity assumption on $f$ and $\phi, F: \mathrm{R}^{+} \times$ $\mathrm{X} \rightarrow \mathrm{X}$ is $C^{2}$ smooth.

We want to show, by using the Crandal-Rabinowitz Theorem, that a local bifurcation of solutions of $(5.9)$ occurs at $\left(\chi_{2},(u, v, w)\right)=\left(\chi\left(\lambda_{2}\right),(0,0,0)\right)$.

To this end, we have to show

(i) $\operatorname{dim} N\left(F_{(u, v, w)}\left(\chi\left(\lambda_{2}\right),(0,0,0)\right)\right)=1=\operatorname{codim} \mathrm{R}\left(F_{(u, v, w)}\left(\chi\left(\lambda_{2}\right),(0,0,0)\right)\right)$.

(ii) $F_{\chi_{2}(u, v, w)}\left(\chi\left(\lambda_{2}\right),(0,0,0)\right)\left(u_{0}, v_{0}, w_{0}\right) \notin \mathrm{R}\left(F_{(u, v, w)}\left(\chi\left(\lambda_{2}\right),(0,0,0)\right)\right)$, where $\left(u_{0}, v_{0}, w_{0}\right)$ spans $N\left(F_{(u, v, w)}\left(\chi\left(\lambda_{2}\right),(0,0,0)\right)\right)$.

Since $F_{(u, v, w)}\left(\chi\left(\lambda_{2}\right),(0,0,0)\right)(u, v, w)$ is the linear parts of (5.2), (5.7), and (5.8), $\left(u_{0}, v_{0}, w_{0}\right)$ satisfies $(4.3)$ with $\eta=0$ and $\chi_{2}=\chi\left(\lambda_{2}\right)$.

From now on, we assume that $(\bar{u}, \bar{v})$ is locally asymptotically stable in $\mathrm{H}^{1}(0,1)$. Then since $\eta^{*}\left(\lambda_{2}, \chi\left(\lambda_{2}\right)\right)=0$, a nonzero $\left(u_{0}, v_{0}, w_{0}\right)$ exists and the set of such is one dimensional, with $w_{0}$ being the first eigenfunction of the $w$-eigenvalue problem in (4.3) with $\chi_{2}=\chi\left(\lambda_{2}\right)$. Since $F_{(u, v, w)}\left(\chi\left(\lambda_{2}\right),(0,0,0)\right)$ is a Fredholm operator with 0 index, (i) is verified.

Now we verify (ii). Observe that

$$
F_{\chi_{2}(u, v, w)}\left(\chi\left(\lambda_{2}\right),(0,0,0)\right)\left(u_{0}, v_{0}, w_{0}\right)=\left(0,0, K_{3}\left[\left(\bar{u}^{\prime} \phi^{\prime}(\bar{u}) w_{0}\right)^{\prime}\right]+B_{3}\left(w_{0}\right)\right)
$$


If this is equal to $F_{(u, v, w)}\left(\chi\left(\lambda_{2}\right),(0,0,0)\right)(u, v, w)$, then $w-K_{3}[(k f(\bar{u})-\theta) w+w]=$ $K_{3}\left[\left(\bar{u}^{\prime} \phi^{\prime}(\bar{u}) w_{0}\right)^{\prime}\right]+B_{3}\left(w_{0}\right)$, and hence

$$
\left\{\begin{array}{cl}
\left(\lambda_{2} w^{\prime}-\chi\left(\lambda_{2}\right) \bar{u}^{\prime} \phi^{\prime}(\bar{u}) w\right)^{\prime}+(k f(\bar{u})-\theta) w & \\
=-\left(\bar{u}^{\prime} \phi^{\prime}(\bar{u}) w_{0}\right)^{\prime}, & x \in(0,1), \\
\lambda_{2} w^{\prime}-\chi\left(\lambda_{2}\right) \bar{u}^{\prime} \phi^{\prime}(\bar{u}) w=-\bar{u}^{\prime} \phi^{\prime}(\bar{u}) w_{0}, & x=0,1 .
\end{array}\right.
$$

Let $z_{2}=w e^{-\chi\left(\lambda_{2}\right) \phi(\bar{u}) / \lambda_{2}}, W_{0}=w_{0} e^{-\chi\left(\lambda_{2}\right) \phi(\bar{u}) / \lambda_{2}}$. Then they satisfy

$$
\begin{aligned}
& \begin{cases}\left(\lambda_{2} z_{2}^{\prime} e^{\chi\left(\lambda_{2}\right) \phi(\bar{u}) / \lambda_{2}}\right)^{\prime}+(k f(\bar{u})-\theta) z_{2} e^{\chi\left(\lambda_{2}\right) \phi(\bar{u}) / \lambda_{2}} & \\
=-\left(\bar{u}^{\prime} \phi^{\prime}(\bar{u}) w_{0}\right)^{\prime}, & x \in(0,1), \\
\lambda_{2} z_{2}^{\prime} e^{\chi\left(\lambda_{2}\right) \phi(\bar{u}) / \lambda_{2}}=-\bar{u}^{\prime} \phi^{\prime}(\bar{u}) w_{0}, & x=0,1 .\end{cases} \\
& \begin{cases}\left(\lambda_{2} W_{0}^{\prime} e^{\chi\left(\lambda_{2}\right) \phi(\bar{u}) / \lambda_{2}}\right)^{\prime}+(k f(\bar{u})-\theta) W_{0} e^{\chi\left(\lambda_{2}\right) \phi(\bar{u}) / \lambda_{2}}=0, & x \in(0,1), \\
W_{0}^{\prime}=0, & x=0,1 .\end{cases}
\end{aligned}
$$

Multiplying (5.11) by $W_{0}$ and (5.12) by $z_{2}$ and integrating by parts, we obtain

$$
\begin{gathered}
-\int_{0}^{1}\left(\bar{u}^{\prime} \phi^{\prime}(\bar{u}) w_{0}\right)^{\prime} W_{0} d x=\left.\lambda_{2} z_{2}^{\prime} e^{\chi\left(\lambda_{2}\right) \phi(\bar{u}) / \lambda_{2}} W_{0}\right|_{0} ^{1} \\
\int_{0}^{1} \bar{u}^{\prime} \phi^{\prime}(\bar{u}) w_{0} W_{0}^{\prime} d x-\left.\bar{u}^{\prime} \phi^{\prime}(\bar{u}) w_{0} W_{0}\right|_{0} ^{1}=-\left.\bar{u}^{\prime} \phi^{\prime}(\bar{u}) w_{0} W_{0}\right|_{0} ^{1} \\
\int_{0}^{1} \bar{u}^{\prime} \phi^{\prime}(\bar{u}) w_{0} W_{0}^{\prime} d x=0 .
\end{gathered}
$$

This is impossible because $w_{0}$ and $W_{0}^{\prime}$ are of one sign; thus (ii) is verified.

Now the following theorem follows from $[\mathrm{CR}]$.

THEOREM 5.1. Suppose $(\bar{u}, \bar{v})$ is locally exponentially asymptotically stable with respect to single species dynamics. For each $\lambda_{2}>\lambda_{0}$, there exists an $\varepsilon>0$ and $C^{1}$ smooth functions $\chi_{2}:\left(-\varepsilon_{1}, \varepsilon_{1}\right) \rightarrow \mathrm{R},\left(\psi_{1}, \psi_{2}, \psi_{3}\right):\left(-\varepsilon_{1}, \varepsilon_{1}\right) \rightarrow \mathrm{Z}$, where $\mathrm{Z}$ is a complement of $\operatorname{span}\left(u_{0}, v_{0}, w_{0}\right)$ in $\mathrm{X}^{3}$, such that $\chi_{2}(0)=\chi\left(\lambda_{2}\right), \psi_{1}(0)=0=\psi_{2}(0)=\psi_{3}(0)$ and such that for $r \in\left(-\varepsilon_{1}, \varepsilon_{1}\right), \tilde{u}(r)=r\left(u_{0}+\psi_{1}(r)\right), \tilde{v}(r)=r\left(v_{0}+\psi_{2}(r)\right)$, and $\tilde{w}(r)=r\left(w_{0}+\psi_{3}(r)\right)$ satisfy (5.9). Moreover, all solutions of (5.9) near $\left(\chi\left(\lambda_{2}\right),(0,0,0)\right)$ are either on the curve $\left(\chi_{2}(r),(\tilde{u}(r), \tilde{v}(r), \tilde{w}(r))\right.$ or on $(u, v, w)=0$.

REMARK 5.2. By taking a positive $w_{0}$ (which we do from now on) and a small $\varepsilon_{1}$, we see $\tilde{w}(r)>0$ on $[0,1]$ for all $0<r<\varepsilon_{1}$. Thus $(\bar{u}+\tilde{u}(r), \bar{v}+\tilde{v}(r), \tilde{w}(r))$ is a positive steady state of (1.3) with $\chi_{2}=\chi_{2}(r)$ if $0<r<\varepsilon_{1}$.

We now want to extend the local bifurcation curve to a global one. Let $C$ be the maximum subcontinuum of the closure of the set of solutions of $(5.9)$ with $(u, v, w) \neq$ $(0,0,0)$, passing through $\left(\chi\left(\lambda_{2}\right),(0,0,0)\right)$. Let $C^{+}$be the maximum subcontinuum of the closure of $C \backslash\left\{\left(\chi_{2}(r),(\tilde{u}(r), \tilde{v}(r), \tilde{w}(r)) \mid-\varepsilon_{1}<r<0\right\}\right.$. Then by combining the reflection arguments in $\left[\mathrm{R}\right.$, Theorem 1.27] and [BB, Theorem 3.2], we have that $C^{+}$ either meets "infinity" or meets $(\hat{\chi},(0,0,0))$, where $\hat{\chi} \neq \chi\left(\lambda_{2}\right)$ and $F_{(u, v, w)}(\hat{\chi},(0,0,0))$ is 
not invertible, or $C^{+}$contains a pair of points $(\chi,(u, v, w)$,$) and (\chi,-(u, v, w))$, provided the following condition is met: there exists a small $\delta>0$ such that

$$
\begin{aligned}
\text { index }\left(F\left(\chi\left(\lambda_{2}\right)-\delta,(u, v, w)\right),(0,0,0)\right) & \\
& \neq \text { index }\left(F\left(\chi\left(\lambda_{2}\right)+\delta,(u, v, w)\right),(0,0,0)\right) .
\end{aligned}
$$

To prove this, we observe that $F_{(u, v, w)}\left(\chi_{2},(0,0,0)\right)=I-T$, where $T$ is a linear compact operator, and hence

$$
\text { index }\left(F\left(\chi_{2},(u, v, w)\right),(0,0,0)\right)=(-1)^{p},
$$

where $p$ is the sum of the algebraic multiplicities of the real eigenvalues of $T$ that are greater than 1 .

LEMmA 5.3. There exists a small $\delta_{0}>0$ such that if $k f(1)-\delta_{0}<\theta<k f(1)$, then $p=0$ for any $0 \leq \chi_{2}<\chi\left(\lambda_{2}\right), \lambda_{0}<\lambda_{2}$.

Proof. Let $\eta>1$ be an eigenvalue of $T$ in $\mathrm{X}^{3}$ and $(\hat{u}, \hat{v}, \hat{w})$ be a corresponding eigenvector. Then

$$
\left\{\begin{array}{l}
-K_{1}\left[f(\bar{u})+(\hat{v}+\hat{w})+f^{\prime}(\bar{u}) \bar{v} \hat{u}\right]=\eta \hat{u}, \\
K_{2} \hat{v}-\chi_{1} K_{2}\left[\left(\bar{v} \phi^{\prime}(\bar{u})\right)^{\prime} \hat{u}^{\prime}+\left(\bar{u}^{\prime} \bar{v} \phi^{\prime \prime}(\bar{u}) \hat{u}\right)^{\prime}+\bar{v} \phi^{\prime}(\bar{u})(f(\bar{u})(\hat{v}+\hat{w})\right. \\
\left.\left.\quad+f^{\prime}(\bar{u}) \bar{v} \hat{u}\right)\right]+K_{2}\left[k f^{\prime}(\bar{u}) \bar{v} \hat{u}+(k f(\bar{u})-\theta) \hat{v}\right]-B_{1}(\hat{u})=\eta \hat{v}, \\
K_{3}[(k f(\bar{u})-\theta) \hat{w}+\hat{w}]-\left(\chi_{2}-\chi\left(\lambda_{2}\right)\right) K_{3}\left[\left(\bar{u}^{\prime} \phi^{\prime}(\bar{u}) \hat{w}\right)^{\prime}\right] \\
\quad\left(\chi_{2}-\chi\left(\lambda_{2}\right)\right) B_{3}(\hat{w})=\eta \hat{w} .
\end{array}\right.
$$

In particular,

$$
\left\{\begin{array}{cc}
\left(\eta \lambda_{2} \hat{w}^{\prime}-\left(\chi_{2}+(\eta-1) \chi\left(\lambda_{2}\right)\right) \bar{u}^{\prime} \phi^{\prime}(\bar{u}) \hat{w}\right)^{\prime} & \\
+(k f(\bar{u})-\theta) \hat{w}+(1-\eta) \hat{w}=0, & x \in(0,1) \\
\eta \lambda_{2} \hat{w}^{\prime}-\left(\chi_{2}+(\eta-1) \chi\left(\lambda_{2}\right)\right) \bar{u}^{\prime} \phi^{\prime}(\bar{u}) \hat{w}=0, & x=0,1 .
\end{array}\right.
$$

Let $a=\frac{\chi_{2}+(\eta-1) \chi\left(\lambda_{2}\right)}{\eta}, \quad \hat{z}=e^{-a \phi(\bar{u}) / \lambda_{2}} \hat{w}$. Then

$$
\left\{\begin{array}{l}
\lambda_{2} \eta\left(\hat{z}^{\prime} e^{a \phi(\bar{u}) / \lambda_{2}}\right)^{\prime}+(k f(\bar{u})-\theta+1-\eta) \hat{z} e^{a \phi(\bar{u}) / \lambda_{2}}=0, \quad x \in(0,1), \\
\hat{z}^{\prime}(0)=0=\hat{z}^{\prime}(1) .
\end{array}\right.
$$

Multiplying both sides by $\hat{z}$ and integrating by parts, we obtain

$$
\begin{aligned}
0 & =\int_{0}^{1}\left[\eta \lambda_{2}\left(\hat{z}^{\prime}\right)^{2}-(k f(\bar{u})-\theta+1-\eta) \hat{z}^{2}\right] e^{a \phi(\bar{u}) / \lambda_{2}} d x \\
& \geq \int_{0}^{1}\left[\lambda_{2}\left(\hat{z}^{\prime}\right)^{2}-(k f(\bar{u})-\theta) \hat{z}^{2}\right] e^{a \phi(\bar{u}) / \lambda_{2}} d x \\
& \geq-\eta^{*}\left(\lambda_{2}, a\right) \int_{0}^{1} \hat{z}^{2} e^{a \phi(\bar{u}) / \lambda_{2}} d x \geq 0,
\end{aligned}
$$

because $a<\chi\left(\lambda_{2}\right)$ and hence by Lemmas 4.2 and $4.5, \eta^{*}\left(\lambda_{2}, a\right)<0$. Thus $\hat{z} \equiv 0$. Now $(\hat{u}, \hat{v}) \neq(0,0)$. But by the proof of Theorem 5.2 in [W], $\eta<1$ if $\theta$ is close to $k f(1)$ (the closeness is independent of $\chi_{2}$ and $\lambda_{2}$ ). This contradiction completes the proof of Lemma 5.3. 
Lemma 5.4. For each $\theta \in\left(k f(1)-\delta_{0}, k f(1)\right), p=1$ if $\chi_{2}$ is bigger and close to $\chi\left(\lambda_{2}\right)$.

Proof. As mentioned in the proof of Lemma 5.3, (5.14) has no solution with $\eta>1$ and $\hat{w}=0$. Thus to show $p=1$, we only need to show that the third component of $T$, denoted by $T^{3}$, has only one eigenvalue bigger than 1 , which is also simple. $T^{3}$ : $\mathrm{X} \rightarrow \mathrm{X}$ is linear and compact, depending continuously on $\chi_{2} . \eta=1$ is an eigenvalue of $\left.T^{3}\right|_{\chi_{2}=\chi\left(\lambda_{2}\right)}$ with the corresponding eigenspace spanned by $w_{0}>0$. This eigenvalue of $\left.T^{3}\right|_{\chi_{2}=\chi\left(\lambda_{2}\right)}$ is simple, as can be proved as follows. Suppose there exists $w$ such that $\left(\left.T^{3}\right|_{\chi_{2}=\chi\left(\lambda_{2}\right)}-I\right) w=w_{0}$. Then we have

$$
\left\{\begin{array}{cl}
\left(\lambda_{2} w^{\prime}-\chi\left(\lambda_{2}\right) \bar{u}^{\prime} \phi^{\prime}(\bar{u}) w\right)^{\prime}+(k f(\bar{u})-\theta) w & \\
=\left(-\lambda_{2} w_{0}^{\prime}+\chi\left(\lambda_{2}\right) \bar{u}^{\prime} \phi^{\prime}(\bar{u}) w_{0}\right)^{\prime}+w_{0}, & x \in(0,1), \\
\lambda_{2} w^{\prime}-\chi\left(\lambda_{2}\right) \bar{u}^{\prime} \phi^{\prime}(\bar{u}) w=0, & x=0,1 .
\end{array}\right.
$$

Define $z=w e^{-\chi\left(\lambda_{2}\right) \phi(\bar{u}) / \lambda_{2}}$. Then

$$
\left\{\begin{array}{l}
\lambda_{2}\left(z^{\prime} e^{\chi\left(\lambda_{2}\right) \phi(\bar{u}) / \lambda_{2}}\right)^{\prime}+(k f(\bar{u})-\theta) z e^{\chi\left(\lambda_{2}\right) \phi(\bar{u}) / \lambda_{2}} \\
\quad=-\lambda_{2}\left(W_{0}^{\prime} e^{\chi\left(\lambda_{2}\right) \phi(\bar{u}) / \lambda_{2}}\right)^{\prime}+W_{0} e^{\chi\left(\lambda_{2}\right) \phi(\bar{u}) / \lambda_{2}}, \quad x \in(0,1), \\
z^{\prime}(0)=0=z^{\prime}(1)
\end{array}\right.
$$

where $W_{0}$ is as defined above (5.11).

Multiplying (5.18) by $W_{0}$ and $(5.12)$ by $z$, we obtain $W_{0}=0$, which is impossible. Therefore, $\eta=1$ is a simple eigenvalue of $\left.T^{3}\right|_{\chi_{2}=\chi\left(\lambda_{2}\right)}$.

By Lemma 1.3 of $[\mathrm{CR}]$, for $\chi_{2}$ close to $\chi\left(\lambda_{2}\right)$, in a small disk $D_{r}(1)$ on the complex plane centered at 1 with radius $r, T^{3}$ has only one eigenvalue $\eta_{1}$ which must also be simple. Since $T^{3}$ is real, $\eta_{1}$ must also be real (otherwise, the conjugate of $\eta_{1}$ is also an eigenvalue in $\left.D_{r}(1)\right)$. We now show $\eta_{1}>1$. Let $\hat{w}$ be a corresponding eigenfunction of $T^{3}$, normalized by $\|\hat{w}\|_{L^{2}(0,1)}=\left\|w_{0}\right\|_{L^{2}(0,1)}$. Then it is easy to see that $\hat{w} \rightarrow \pm w_{0}$ in $C^{1}[0,1]$ as $\chi_{2} \rightarrow \chi\left(\lambda_{2}\right)$. So we may assume, without loss of generality, that $\hat{w}>0$. Define $\hat{z}$ as in the proof of Lemma 5.3 (with $\eta=\eta_{1}$ ). Then by (5.16), (5.12), and the proof of Theorem 3.1, we have $\eta_{1}>1$. Since $\left.T^{3}\right|_{\chi_{2}=\chi\left(\lambda_{2}\right)}$ has no real eigenvalue bigger than $1+r$ (see the argument below (5.16)), the same is true for $T^{3}$ if $\chi_{2}$ is close to $\chi\left(\lambda_{2}\right)$. This proves $p=1$.

Now (5.13) follows from Lemmas 5.3 and 5.4.

For any $\left(\chi_{2},(\tilde{u}, \tilde{v}, \tilde{w})\right) \in C^{+}$, let $u=\bar{u}+\tilde{u}, v=\bar{v}+\tilde{v}$. Then $(u, v, \tilde{w})$ satisfies (3.1), except that we do not know at this point that it is nonnegative. Define

$$
S^{+}=\left\{\left(\chi_{2},(u, v, \tilde{w})\right) \mid\left(\chi_{2},(\tilde{u}, \tilde{v}, \tilde{w})\right) \in C^{+}\right\} \backslash\left\{\left(\chi\left(\lambda_{2}\right),(\bar{u}, \bar{v}, 0)\right)\right\} .
$$

Then $S^{+}$is a continuum in $\mathrm{R} \times \mathrm{X}^{3}$, either meeting "infinity", or $(\hat{\chi},(\bar{u}, \bar{v}, 0))$ with $\hat{\chi} \neq$ $\chi\left(\lambda_{2}\right)$, or containing a pair of points $\left(\chi_{2},(\bar{u}+\tilde{u}, \bar{v}+\tilde{v}, \tilde{w})\right)$ and $\left(\chi_{2},(\bar{u}-\tilde{u}, \bar{v}-\tilde{v},-\tilde{w})\right)$.

Define

$$
P^{+}=\left\{\left(\chi_{2},(u, v, w)\right) \in \mathrm{R} \times \mathrm{X}^{3} \mid \chi_{2} \geq 0,1>u>0, v>0, w>0\right\} .
$$

LEMMA 5.5. $S^{+}$is not entirely contained in $P^{+}$(but of course the part of $S^{+}$near $\left(\chi\left(\lambda_{2}\right),(\bar{u}, \bar{v}, 0)\right)$ is $)$. 
Proof. Otherwise, $S^{+}$meets infinity and any $\left(\chi_{2},(u, v, \tilde{w})\right)$ in $S^{+}$is a positive solution of (3.1). It is easy to show that $\|(u, v, \tilde{w})\|_{\mathrm{X}^{3}}$ is bounded for bounded $\chi_{2} \geq 0$. This forces the projection of $S^{+}$on the $\chi_{2}$-axis to cover the interval $\left(\chi\left(\lambda_{2}\right), \infty\right)$, which means $(3.1)$ has a positive solution for every $\chi_{2}>\chi\left(\lambda_{2}\right)$, contradicting Theorem 3.3. This proves Lemma 5.5.

By this result, $S^{+} \cap \partial P^{+}$is nonempty. For any $\left(\chi_{2},(u, v, \tilde{w})\right) \in S^{+} \cap \partial P^{+}$, there exists a sequence $\left(\chi_{2}^{n},\left(u^{n}, v^{n}, \tilde{w}^{n}\right)\right)$ in $S^{+} \cap P^{+}$which converges to it as $n \rightarrow \infty$. We claim that

$$
1>u>0, v \equiv 0 \quad \text { and } \quad \tilde{w}>0 \quad \text { on }[0,1], \quad \text { for }\left(\chi_{2},(u, v, \tilde{w})\right) \in S^{+} \cap \partial P^{+} .
$$

First we show $1>u>0$. Otherwise, $1 \geq u \geq 0$ and $u=0$ or 1 somewhere on $[0,1]$. Then the strong maximum principle and Hopf boundary point lemma imply $u \equiv 1$ or 0 . $u \equiv 0$ is impossible because of the boundary condition at $x=1 . u \equiv 1$ is also impossible because $\int_{0}^{1}\left(k f\left(u^{n}\right)-\theta\right) \tilde{w}^{n} d x$, which is 0 , would be positive for large $n$.

Now we show $\tilde{w}>0$ on $[0,1]$. Otherwise, $\tilde{w} \equiv 0$ on $[0,1]$. Then $v$ must be positive on $[0,1]$, for if not, $v \equiv 0$. Then $u^{\prime \prime} \equiv 0$ and by the boundary condition, $u \equiv 1$ which is again impossible. Now by the uniqueness of the positive solution of (3.1) with $w \equiv 0$ for $\theta$ close to $k f(1)$ (see $[\mathrm{W}]), u=\bar{u}, v=\bar{v}$ and hence $\tilde{u}=0=\tilde{v}$. Then $\left(\tilde{u}^{n}, \tilde{v}^{n}, \tilde{w}^{n}\right)=\left(u^{n}-\bar{u}, v^{n}-\bar{v}, \tilde{w}^{n}\right) \rightarrow 0$ as $n \rightarrow \infty$. Now in (5.9), substitute $\left(\chi_{2},(u, v, w)\right)$ by $\left(\chi_{2}^{n},\left(\tilde{u}^{n}, \tilde{v}^{n}, \tilde{w}^{n}\right)\right)$, then divide it by $\left\|\left(\tilde{u}^{n}, \tilde{v}^{n}, \tilde{w}^{n}\right)\right\|_{\mathrm{X}^{3}}$. After passing to a subsequence, $\left(\tilde{u}^{n}, \tilde{v}^{n}, \tilde{w}^{n}\right) /\left\|\left(\tilde{u}^{n}, \tilde{v}^{n}, \tilde{w}^{n}\right)\right\|_{\mathrm{X}^{3}} \rightarrow\left(\tilde{u}_{\infty}, \tilde{v}_{\infty}, \tilde{w}_{\infty}\right)$, where $\left\|\left(\tilde{u}_{\infty}, \tilde{v}_{\infty}, \tilde{w}_{\infty}\right)\right\|_{\mathrm{X}^{3}}=1$, $\tilde{w}_{\infty} \geq 0$ on $[0,1]$ and $F_{(u, v, w)}\left(\chi_{2},(0,0,0)\right)\left(\tilde{u}_{\infty}, \tilde{v}_{\infty}, \tilde{w}_{\infty}\right)=0$. If $\tilde{w}_{\infty} \equiv 0$, then as before, $\left(\tilde{u}_{\infty}, \tilde{v}_{\infty}\right)=(0,0)$, which is impossible. Thus $\tilde{w}_{\infty}>0$ on $[0,1]$. This is only possible if $\chi_{2}=\chi\left(\lambda_{2}\right)$, which is impossible by the definition of $S^{+}$. Now $v \equiv 0$ on $[0,1]$. This completes the proof of $(5.19)$.

Combining Theorem 5.1, Lemmas 5.3-5.5, and (5.19), we have

THEOREM 5.6. There exists a small $\delta_{0}>0$ such that if $k f(1)-\delta_{0}<\theta<k f(1)$, then for every $\lambda_{2}>\lambda_{0},(3.1)$ has a continuum of positive solutions $\left(\chi_{2},(u, v, w)\right)$, joining the semitrivial solutions $\left(\chi\left(\lambda_{2}\right),(\bar{u}, \bar{v}, 0)\right)$ and $\left(\chi_{2}^{\infty},(\underline{u}, 0, \underline{w})\right)$.

REMARK 5.7. If $\chi\left(\lambda_{2}\right)=\chi_{1}$, then by Theorem 3.1 and Remark 3.2 , the continuum of positive solutions of $(3.1)$ mentioned above is just $\left(\chi_{1},(\bar{u}, \alpha \bar{v},(1-\alpha) \bar{v})\right)$, where $0<\alpha<1$.

6. Stability of bifurcating solutions. In this section, we prove the local stability of the bifurcating positive steady states $(u, v, w)=(\bar{u}+\tilde{u}, \bar{v}+\tilde{v}, \tilde{w})$ of $(1.3)$ (see Theorem 5.1 for notation), for $\theta$ close to $k f(1)$ and $\lambda_{1} \neq \lambda_{2}$. To this end, we first need to show that the bifurcation curve is "tilted to the right", i.e., $\left.\frac{d \chi_{2}(r)}{d r}\right|_{r=0}>0$.

LEMMA 6.1.

$$
\begin{aligned}
& \left.\frac{d \chi_{2}(r)}{d r}\right|_{r=0} \\
& =\frac{\int_{0}^{1} u_{0} e^{\chi\left(\lambda_{2}\right) \phi(\bar{u}) / \lambda_{2}}\left[-k f^{\prime}(\bar{u}) W_{0}^{2}+\chi\left(\lambda_{2}\right)\left(W_{0}^{\prime}\right)^{2} \phi^{\prime}(\bar{u})-\frac{\chi\left(\lambda_{2}\right)}{\lambda_{2}}(k f(\bar{u})-\theta) W_{0}^{2} \phi^{\prime}(\bar{u})\right] d x}{\int_{0}^{1} \bar{u}^{\prime} \phi^{\prime}(\bar{u}) w_{0} W_{0}^{\prime} d x},
\end{aligned}
$$

where $W_{0}$ is as defined above (5.11). 
Proof. Define $z_{2}$ as in (3.2) with $\chi_{2}=\chi_{2}(r)$. Multiplying the $z_{2}$-equation in (3.3) by $W_{0} \exp \left(\left(\chi\left(\lambda_{2}\right) \phi(\bar{u})-\chi_{2} \phi(u)\right) / \lambda_{2}\right)$, and (5.12) by $z_{2}$, we obtain

$$
\int_{0}^{1} W_{0} e^{\chi\left(\lambda_{2}\right) \phi(\bar{u}) / \lambda_{2}}\left[k(f(u)-f(\bar{u})) z_{2}+z_{2}^{\prime}\left(\chi_{2} \phi^{\prime}(u) u^{\prime}-\chi\left(\lambda_{2}\right) \bar{u}^{\prime} \phi^{\prime}(\bar{u})\right)\right] d x=0 .
$$

Dividing this by $r^{2}$ and sending it to zero, by Theorem 5.1 , we have

$$
\begin{aligned}
\int_{0}^{1} k f^{\prime}(\bar{u}) u_{0} W_{0}^{2} e^{\chi\left(\lambda_{2}\right) \phi(\bar{u}) / \lambda_{2}} d x+\frac{d \chi_{2}}{d r}(0) \int_{0}^{1} W_{0}^{\prime} W_{0} \bar{u}^{\prime} \phi^{\prime}(\bar{u}) e^{\chi\left(\lambda_{2}\right) \phi(\bar{u}) / \lambda_{2}} d x \\
+\chi\left(\lambda_{2}\right) \int_{0}^{1} W_{0}^{\prime} W_{0}\left(\phi^{\prime}(\bar{u}) u_{0}^{\prime}+\bar{u}^{\prime} \phi^{\prime \prime}(\bar{u}) u_{0}\right) e^{\chi\left(\lambda_{2}\right) \phi(\bar{u}) / \lambda_{2}} d x=0 .
\end{aligned}
$$

Multiplying (5.12) by $\phi^{\prime}(\bar{u}) u_{0} W_{0}$, integrating by parts and combining the resulting equation with (6.1), we conclude the proof of Lemma 6.1.

The bulk of the rest of this section is to show that $\left.\frac{d \chi_{2}(r)}{d r}\right|_{r=0}>0$ for fixed $\lambda_{1}, \chi_{1}$ and $\lambda_{2}>\lambda_{0}$ if $\theta$ is close enough to $k f(1)$. Recall from [Z] and [W] that there exists a positive $\delta_{1}>0$ and $C^{1}$ smooth functions $\mu: s \in\left[-\delta_{1}, \delta_{1}\right] \rightarrow \mathrm{R},\left(\phi_{1}, \phi_{2}\right): s \in\left[-\delta_{1}, \delta_{1}\right] \rightarrow \mathrm{Y}$ such that $\mu(0)=0, \mu^{\prime}(0)>0, \phi_{1}(0)=\phi_{2}(0)=0$ and $(\bar{u}, \bar{v})=\left(1+s\left(u^{*}+\phi_{1}(s)\right), s\left(1+\phi_{2}(s)\right)\right)$, $s \in\left(0, \delta_{1}\right]$, is the unique positive solution of $(3.1)$ with $w=0$ and $\theta=k f(1)-\mu(s)$, where $\mathrm{Y}$ is any complement of $\operatorname{span}\left(u^{*}, 1\right)$ in $\mathrm{X} \times \mathrm{X}$, which is taken to be $\mathrm{Y}=\{(u, v) \in$ $\left.\mathrm{X} \times \mathrm{X} \mid \int_{0}^{1} u^{*} u d x=0=\int_{0}^{1} v d x\right\}, u^{*}=-K_{1} f(1)=\frac{1}{2} f(1)\left(x^{2}-1\right)-\frac{1}{h} f(1)$. Recall also that

$$
\mu^{\prime}(0)=-k f^{\prime}(1) \int_{0}^{1} u^{*} d x\left(=k f^{\prime}(1) f(1)(1 / 3+1 / h)\right) .
$$

Lemma 6.2. (i) $\lim _{s \rightarrow 0^{+}} \lambda_{0}=\frac{2 \lambda_{1} k f^{\prime}(1)}{2 k f^{\prime}(1)+21 \chi_{1} \phi^{\prime}(1)}$ (denote this by $\left.\lambda_{0}^{0}\right)$.

(ii) For bounded $\lambda_{2}>\lambda_{0}^{0}, \chi\left(\lambda_{2}\right)$ remains bounded as $s \rightarrow 0^{+}$.

Proof. By the definition of $\lambda_{0}$ (see $\left.(4.8)\right), \eta^{*}\left(\lambda_{0}, 0\right)=0$, i.e.,

$$
0=\inf _{\substack{z \in \mathrm{H}^{1}(0,1) \\ z \neq 0}} \frac{\int_{0}^{1}\left[\lambda_{0}\left(z^{\prime}\right)^{2}-(k f(\bar{u})-\theta) z^{2}\right] d x}{\int_{0}^{1} z^{2} d x}
$$

and hence

$$
\lambda_{0} \geq \sup _{\substack{z \in \mathrm{H}^{1}(0,1) \\ z \not \equiv 0}} \frac{\int_{0}^{1}(k f(\bar{u})-\theta) z^{2} d x}{\int_{0}^{1}\left(z^{\prime}\right)^{2} d x}
$$

In particular,

$$
\lambda_{0} \geq \frac{\int_{0}^{1}(k f(\bar{u})-\theta) \bar{V}^{2} e^{\chi_{1} \phi(\bar{u}) / \lambda_{1}} d x}{\int_{0}^{1}\left(\bar{V}^{\prime}+\frac{\chi_{1}}{2 \lambda_{1}} \bar{u}^{\prime} \phi^{\prime}(\bar{u}) \bar{V}\right)^{2} e^{\chi_{1} \phi(\bar{u}) / \lambda_{1}} d x}
$$

where $\bar{V}=\bar{v} e^{-\chi_{1} \phi(\bar{u}) / \lambda_{1}}$, which satisfies

$$
\left\{\begin{array}{l}
\lambda_{1}\left(\bar{V}^{\prime} e^{\chi_{1} \phi(\bar{u}) / \lambda_{1}}\right)^{\prime}+(k f(\bar{u})-\theta) \bar{V} e^{\chi_{1} \phi(\bar{u}) / \lambda_{1}}=0, \quad x \in(0,1), \\
\bar{V}^{\prime}(0)=0=\bar{V}^{\prime}(1)
\end{array}\right.
$$


Since

$$
\lambda_{1} \bar{V}^{\prime} e^{\chi_{1} \phi(\bar{u}) / \lambda_{1}}=-\int_{0}^{x}(k f(\bar{u})-\theta) \bar{v}(y) d y,
$$

we have that uniformly for $x \in[0,1]$,

$$
\lim _{s \rightarrow 0^{+}} \frac{\bar{V}^{\prime}}{s^{2}}=-\frac{1}{\lambda_{1}} e^{-\chi_{1} \phi(1) / \lambda_{1}} F_{1}(x),
$$

where

$$
\begin{aligned}
& F_{1}(x)=\int_{0}^{x} f_{1}(y) d y \\
& f_{1}(x)=\left.\frac{d}{d s}(k f(\bar{u})-\theta)\right|_{s=0}=k f^{\prime}(1) u^{*}(x)+\mu^{\prime}(0) .
\end{aligned}
$$

Notice that $F_{1}(x)=k f^{\prime}(1) f(1)\left(x^{3}-x\right) / 6$. Multiplying (6.6) by $\bar{V}$ and integrating by parts, we see that the numerator on the right-hand side of (6.5) is just $\lambda_{1} \int_{0}^{1}\left(\bar{V}^{\prime}\right)^{2} e^{\chi_{1} \phi(\bar{u}) / \lambda_{1}} d x$. Now using (6.8), we have

$$
\liminf _{s \rightarrow 0^{+}} \lambda_{0} \geq \frac{\lambda_{1} \int_{0}^{1} F_{1}^{2}(x) d x}{\int_{0}^{1}\left(-F_{1}(x)+\frac{\chi_{1}}{2} \phi^{\prime}(1)\left(u^{*}\right)^{\prime}\right)^{2} d x}>0 .
$$

Observe that $\lambda_{0} \leq \lambda_{1}$. So for any sequence $s \rightarrow 0^{+}$, there exists a subsequence such that along this subsequence, $\lambda_{0} \rightarrow \lambda_{0}^{0}$, which is positive according to (6.10). Let $z$ be the (positive) minimizer of (6.3), normalized by $\int_{0}^{1} z d x=1$. Then it satisfies

$$
\left\{\begin{array}{l}
\lambda_{0} z^{\prime \prime}+(k f(\bar{u})-\theta) z=0, \quad x \in(0,1) \\
z^{\prime}(0)=0=z^{\prime}(1)
\end{array}\right.
$$

It is easy to see that $z(x) \rightarrow 1$ in $C^{0}[0,1]$ and that

$$
\frac{z^{\prime}(x)}{s} \rightarrow \frac{-1}{\lambda_{0}^{0}} \int_{0}^{x} f_{1}(y) d y, \quad \text { in } C^{0}[0,1] \text { as } s \rightarrow 0^{+} \text {. }
$$

By (6.11) and (6.6), we have

$$
\lambda_{0} \int_{0}^{1} z^{\prime}\left(\bar{V} e^{\chi_{1} \phi(\bar{u}) / \lambda_{1}}\right)^{\prime} d x=\lambda_{1} \int_{0}^{1} \bar{V}^{\prime} z^{\prime} e^{\chi_{1} \phi(\bar{u}) / \lambda_{1}} d x .
$$

Dividing this by $s^{3}$ and sending $s \rightarrow 0^{+}$, using (6.8) and (6.11), we obtain

$$
\lambda_{0}^{0} \int_{0}^{1} F_{1}(x)\left(F_{1}(x)-\chi_{1}\left(u^{*}\right)^{\prime} \phi^{\prime}(1)\right) d x=\lambda_{1} \int_{0}^{1} F_{1}^{2}(x) d x .
$$

From this and direct computations, (i) follows.

We now prove (ii). For $\lambda_{0} \leq \lambda_{2} \leq \lambda_{1}$, we have $\chi\left(\lambda_{2}\right) \leq \chi_{1}$. So we only need to consider bounded $\lambda_{2}>\lambda_{1}$. By (6.6) and (5.12), we have the following analog of (3.6):

$$
\int_{0}^{1}\left[\lambda_{2}\left(\frac{\chi\left(\lambda_{2}\right)}{\lambda_{2}}-\frac{\chi_{1}}{\lambda_{1}}\right) \bar{V} \bar{u}^{\prime} \phi^{\prime}(\bar{u})-\left(\lambda_{2}-\lambda_{1}\right) \bar{V}^{\prime}\right] W_{0}^{\prime} e^{\chi_{1} \phi(\bar{u}) / \lambda_{1}} d x=0 .
$$

Thus somewhere on $(0,1)$, it follows that

$$
\lambda_{2}\left(\frac{\chi\left(\lambda_{2}\right)}{\lambda_{2}}-\frac{\chi_{1}}{\lambda_{1}}\right) \bar{V} \bar{u}^{\prime} \phi^{\prime}(\bar{u}) \leq\left(\lambda_{2}-\lambda_{1}\right) \bar{V}^{\prime} .
$$


By (6.6), we have

$$
\begin{aligned}
\lambda_{1} \bar{V}^{\prime} e^{\chi_{1} \phi(\bar{u}) / \lambda_{1}} & \leq \int_{0}^{x}(k f(\bar{u})-\theta) \bar{V}(y) e^{\chi_{1} \phi(\bar{u}) / \lambda_{1}} d y \\
& \leq k f(1) s x \bar{V}(x) e^{\chi_{1} \phi(\bar{u}) / \lambda_{1}}
\end{aligned}
$$

On the other hand,

$$
\begin{aligned}
\bar{u}^{\prime}(x) & =\int_{0}^{x} f(\bar{u}(y)) \bar{v}(y) d y=\int_{0}^{x} f(\bar{u}(y)) s\left(1+\phi_{2}(s)\right) d y \\
& \geq C_{1} s x
\end{aligned}
$$

where $C_{1}$ is a positive constant, independent of small $s>0$.

Combining (6.14)-(6.16), we have

$$
\frac{\chi\left(\lambda_{2}\right)}{\lambda_{2}}-\frac{\chi_{1}}{\lambda_{1}} \leq \frac{\lambda_{2}-\lambda_{1}}{\lambda_{1} \lambda_{2}} \cdot \frac{k f(1)}{C_{1}} .
$$

This proves (ii).

We now study the limiting behavior of $\left(u_{0}, v_{0}, w_{0}\right)$ as $s \rightarrow 0^{+}$. Recall that it satisfies (4.3) with $\eta=0, \chi_{2}=\chi\left(\lambda_{2}\right)$. We normalize it by $w_{0}>0$ on $[0,1]$ and

$$
\left\|u_{0}\right\|_{L^{2}(0,1)}+\left\|v_{0}\right\|_{L^{2}(0,1)}+\left\|w_{0}\right\|_{L^{2}(0,1)}=2 .
$$

Lemma 6.3. For each fixed $\lambda_{2}>\lambda_{0}^{0},\left(u_{0}, v_{0}, w_{0}\right) \rightarrow(0,-1,1)$ in $C^{2}[0,1]$ as $s \rightarrow 0^{+}$.

Proof. By (4.3) (with $\eta=0$ and $\chi_{2}=\chi\left(\lambda_{2}\right)$ ), we easily see that the $C^{2+\alpha}[0,1]$ norms of $u_{0}, v_{0}$, and $w_{0}$ are bounded as $s \rightarrow 0^{+}$. So after passing to a subsequence, $\left(u_{0}, v_{0}, w_{0}\right) \rightarrow\left(u_{0}^{0}, v_{0}^{0}, w_{0}^{0}\right)$ in $C^{2}[0,1]$, as $s \rightarrow 0^{+}$, where the limiting functions satisfy (6.18), $w_{0}^{0} \geq 0$ on $[0,1]$ and

$$
\begin{cases}\left(u_{0}^{0}\right)^{\prime \prime}=f(1)\left(v_{0}^{0}+w_{0}^{0}\right), & x \in(0,1), \\ \left(u_{0}^{0}\right)^{\prime}(0)=0=\left(u_{0}^{0}\right)^{\prime}(1)+h u_{0}^{0}(1), & x \in(0,1), \\ \left(v_{0}^{0}\right)^{\prime \prime}=0=\left(w_{0}^{0}\right)^{\prime \prime}, & \\ \left(v_{0}^{0}\right)^{\prime}(0)=\left(v_{0}^{0}\right)^{\prime}(1)=0=\left(w_{0}^{0}\right)^{\prime}(0)=\left(w_{0}^{0}\right)^{\prime}(1) . & \end{cases}
$$

Thus $v_{0}^{0}=k_{1}$ (const.), $w_{0}^{0}=k_{2}$ (const.) $\geq 0$.

By the $v$-equation in (4.3), we have

$$
\int_{0}^{1} k f^{\prime}(\bar{u}) \bar{v} u_{0} d x+\int_{0}^{1}(k f(\bar{u})-\theta) v_{0} d x=0 .
$$

Dividing this by $s$ and sending $s \rightarrow 0^{+}$, we have

$$
k f^{\prime}(1) \int_{0}^{1} u_{0}^{0} d x+k_{1} \int_{0}^{1} f_{1}(x) d x=0
$$

and thus $\int_{0}^{1} u_{0}^{0} d x=0$. If $k_{1}+k_{2}>0$, then $0<\left(u_{0}^{0}\right)^{\prime}(x)<\left(u_{0}^{0}\right)^{\prime}(1)$ for $x \in(0,1)$. Then by $(6.19), u_{0}^{0}(1)<0$ and hence $\int_{0}^{1} u_{0}^{0} d x<0$, a contradiction. Similarly, $k_{1}+k_{2}<0$ is impossible. These, (6.18) and (6.19) imply $u_{0}^{0}=0, k_{1}=-1, k_{2}=1$. The proof of Lemma 6.3 is complete. 
Lemma 6.4. For each fixed $\lambda_{2}>\lambda_{0}^{0}, \lim _{s \rightarrow 0^{+}} \chi\left(\lambda_{2}\right)=\chi_{2}^{0}$, where

$$
\lambda_{2}\left(\frac{\chi_{2}^{0}}{\lambda_{2}}-\frac{\chi_{1}}{\lambda_{1}}\right) \phi^{\prime}(1) \int_{0}^{1} F_{1}(x)\left(u^{*}\right)^{\prime} d x+\frac{\lambda_{2}-\lambda_{1}}{\lambda_{1}} \int_{0}^{1} F_{1}^{2}(x) d x=0 .
$$

Proof. By Lemma 6.2, for any sequence $s \rightarrow 0^{+}$, there exists a subsequence $s \rightarrow 0^{+}$ such that $\chi\left(\lambda_{2}\right) \rightarrow \chi_{2}^{0}$.

By (5.12), we have the following analog of (6.8):

$$
\lim _{s \rightarrow 0^{+}} \frac{W_{0}^{\prime}(x)}{s}=-\frac{1}{\lambda_{2}} e^{-\chi_{2}^{0} \phi(1) / \lambda_{2}} F_{1}(x), \quad \text { uniformly for } x \in[0,1] .
$$

Now dividing (6.13) by $s^{3}$ and using (6.8) and (6.21), we have (6.20). This completes the proof of Lemma 6.4 .

LEMma 6.5. For each fixed $\lambda_{2}>\lambda_{0}^{0}$,

$$
\frac{v_{0}-\int_{0}^{1} v_{0}(x) d x}{s} \rightarrow v_{0}^{0}, \quad \frac{w_{0}-\int_{0}^{1} w_{0}(x) d x}{s} \rightarrow w_{0}^{0}, \quad \text { in } C^{2}[0,1] \text { as } s \rightarrow 0^{+},
$$

where

$$
\begin{array}{ll}
\left(v_{0}^{0}\right)^{\prime}(x)=\frac{1}{\lambda_{1}} F_{1}(x)-\frac{\chi_{1}}{\lambda_{1}} \phi^{\prime}(1)\left(u^{*}\right)^{\prime}(x), & \int_{0}^{1} v_{0}^{0}(x) d x=0, \\
\left(w_{0}^{0}\right)^{\prime}(x)=\frac{-1}{\lambda_{2}} F_{1}(x)+\frac{\chi_{2}^{0}}{\lambda_{2}} \phi^{\prime}(1)\left(u^{*}\right)^{\prime}(x), & \int_{0}^{1} w_{0}^{0}(x) d x=0 .
\end{array}
$$

Proof. Let

$$
v_{s}=\frac{v_{0}-\int_{0}^{1} v_{0} d x}{s}, \quad w_{s}=\frac{w_{0}-\int_{0}^{1} w_{0} d x}{s} .
$$

Then $v_{s}$ and $w_{s}$ satisfy

$$
\begin{cases}\left(\lambda_{1} v_{s}^{\prime}-\chi_{1} \frac{\bar{u}^{\prime}}{s} \phi^{\prime}(\bar{u}) v_{0}-\chi_{1} \frac{\bar{v}}{s} \phi^{\prime}(\bar{u}) u_{0}^{\prime}-\chi_{1} \phi^{\prime \prime}(\bar{u}) \bar{u}^{\prime} \frac{\bar{v}}{s} u_{0}\right)^{\prime} & \\ \quad+k \phi^{\prime}(\bar{u}) \frac{\bar{v}}{s} u_{0}+\frac{(k f(\bar{u})-\theta)}{s} v_{0}=0, & x \in(0,1), \\ \lambda_{1} v_{s}^{\prime}-\chi_{1}\left(\frac{\bar{u}}{s}\right)^{\prime} \phi^{\prime}(\bar{u}) v_{0}-\chi_{1} \frac{\bar{v}}{s} \phi^{\prime}(\bar{u}) u_{0}^{\prime}-\chi_{1} \phi^{\prime \prime}(\bar{u}) \bar{u}^{\prime} \frac{\bar{v}}{s} u_{0}=0, & x=0,1, \\ \left(\lambda_{2} w_{s}^{\prime}-\chi\left(\lambda_{2}\right) \phi^{\prime}(\bar{u}) \frac{\bar{u}^{\prime}}{s} w_{0}\right)^{\prime}+\frac{(k f(\bar{u})-\theta)}{s} w_{0}=0, & x \in(0,1), \\ \lambda_{2} w_{s}^{\prime}-\chi\left(\lambda_{2}\right) \phi^{\prime}(\bar{u}) \frac{\bar{u}^{\prime}}{s} w_{0}=0, & x=0,1, \\ \int_{0}^{1} v_{s}(x) d x=0=\int_{0}^{1} w_{s}(x) d x . & \end{cases}
$$

It is easy to see that $\left\|v_{s}\right\|_{C^{2+\alpha}[0,1]}$ and $\left\|w_{s}\right\|_{C^{2+\alpha}[0,1]}$ are bounded as $s \rightarrow 0^{+}$. Therefore, after passing to a subsequence, $v_{s} \rightarrow v_{0}^{0}$ and $w_{s} \rightarrow w_{0}^{0}$ in $C^{2}[0,1]$ as $s \rightarrow 0^{+}$, where the limiting functions satisfy

$$
\begin{cases}\lambda_{1}\left(v_{0}^{0}\right)^{\prime \prime}(x)+\chi_{1}\left(u^{*}\right)^{\prime \prime}(x) \phi^{\prime}(1)-f_{1}(x)=0, & x \in(0,1), \\ \lambda_{1}\left(v_{0}^{0}\right)^{\prime}(x)+\chi_{1}\left(u^{*}\right)^{\prime}(x) \phi^{\prime}(1)=0, & x=0,1, \\ \lambda_{2}\left(w_{0}^{0}\right)^{\prime \prime}(x)-\chi_{2}^{0}\left(u^{*}\right)^{\prime \prime}(x) \phi^{\prime}(1)+f_{1}(x)=0, & x \in(0,1), \\ \lambda_{2}\left(w_{0}^{0}\right)^{\prime}(x)-\chi_{2}^{0}\left(u^{*}\right)^{\prime}(x) \phi^{\prime}(1)=0, & x=0,1, \\ \int_{0}^{1} v_{0}^{0}(x) d x=0=\int_{0}^{1} w_{0}^{0}(x) d x . & \end{cases}
$$

From this we conclude the proof of Lemma 6.5 . 
Similarly, we can use (5.12) and Lemma 6.3 to prove

Lemma 6.6. For any $\lambda_{2}>\lambda_{0}^{0}$,

$$
\lim _{s \rightarrow 0^{+}} \frac{W_{0}-\int_{0}^{1} W_{0}(x) d x}{s}=W_{0}^{0} \quad \text { in } C^{2}[0,1],
$$

where

$$
\left(W_{0}^{0}\right)^{\prime}(x)=-e^{-\chi_{2}^{0} \phi(1) / \lambda_{2}} F_{1}(x) / \lambda_{2}
$$

and

$$
\int_{0}^{1} W_{0}^{0}(x) d x=0
$$

LEMMA 6.7. For every $\lambda_{2}>\lambda_{0}^{0}$,

$$
\lim _{s \rightarrow 0^{+}} \int_{0}^{1} \frac{u_{0}(x)}{s} d x=0 .
$$

Proof. Define $f_{2}(x)$ by

$$
\frac{k f(\bar{u}(x))-\theta}{s}=f_{1}(x)+s f_{2}(x)+o(s) .
$$

By (4.3) with $\eta=0$ and $\chi_{2}=\chi\left(\lambda_{2}\right)$, we have

$$
\begin{aligned}
& \int_{0}^{1} k f^{\prime}(\bar{u}) \frac{\bar{v}}{s} \frac{u_{0}}{s} d x=-\int_{0}^{1} \frac{k f(\bar{u})-\theta}{s} \cdot \frac{w_{0}+v_{0}}{s} d x \\
& \stackrel{\text { Lemma }}{=} 6.5-\int_{0}^{1}\left(f_{1}+s f_{2}+o(s)\right)\left(\frac{1}{s} \int_{0}^{1}\left(v_{0}+w_{0}\right) d x+\left(v_{0}^{0}+w_{0}^{0}\right)+o(1)\right) d x \\
& =-\frac{1}{s} \int_{0}^{1} f_{1} d x \int_{0}^{1}\left(v_{0}+w_{0}\right) d x-\int_{0}^{1} f_{2} d x \int_{0}^{1}\left(v_{0}+w_{0}\right) d x-\int_{0}^{1} f_{1}\left(v_{0}^{0}+w_{0}^{0}\right) d x+o(1) \\
& =\int_{0}^{1} F_{1}\left[\left(\frac{1}{\lambda_{1}}-\frac{1}{\lambda_{2}}\right) \int_{0}^{1} f_{1}+\left(\frac{\chi_{2}^{0}}{\lambda_{2}}-\frac{\chi_{1}}{\lambda_{1}}\right) \phi^{\prime}(1)\left(u^{*}\right)^{\prime}\right] d x \stackrel{(6.20)}{=} 0 .
\end{aligned}
$$

Now we have

$$
\begin{aligned}
\lim _{s \rightarrow 0^{+}} \int_{0}^{1} k f^{\prime}(1) \frac{u_{0}}{s} d x & =\lim _{s \rightarrow 0^{+}} \int_{0}^{1}\left(k f^{\prime}(1)-k f^{\prime}(\bar{u}) \frac{\bar{v}}{s}\right) \frac{u_{0}}{s} d x+\lim _{s \rightarrow 0^{+}} \int_{0}^{1} k f^{\prime}(\bar{u}) \frac{\bar{v} u_{0}}{s^{2}} d x \\
& =-\lim _{s \rightarrow 0^{+}} \int_{0}^{1} \frac{\int_{0}^{s} \frac{d}{d t}\left(k f^{\prime}\left(1+t\left(u^{*}+\phi_{1}(t)\right)\right)\right)\left(1+\phi_{2}(t)\right) d t}{s} u_{0}(x) d x=0
\end{aligned}
$$

because of Lemma 6.3 .

LEMMA 6.8. For every $\lambda_{2}>\lambda_{0}^{0}$,

$$
\lim _{s \rightarrow 0^{+}} \frac{u_{0}}{s}=u_{0}^{0} \quad \text { in } C^{2}[0,1]
$$

where $u_{0}^{0}$ is the unique solution of (6.24) below. 
Proof. Let $u_{s}=u_{0} / s$. By (4.3) with $\eta=0$ and $\chi_{2}=\chi\left(\lambda_{2}\right)$, we have

$$
\begin{gathered}
-h u_{0}(1)=\frac{\theta}{k} \int_{0}^{1}\left(v_{0}+w_{0}\right) d x, \\
\left\{\begin{array}{l}
u_{s}^{\prime \prime}=f^{\prime}(\bar{u}) \bar{v} u_{s}+f(\bar{u})\left(v_{s}+w_{s}\right)-\frac{h k}{\theta} u_{s}(1) f(\bar{u}), \quad x \in(0,1), \\
u_{s}^{\prime}(0)=0=u_{s}^{\prime}(1)+h u_{s}(1) .
\end{array}\right.
\end{gathered}
$$

Multiplying (6.23) by $u_{s}$ and using Lemmas 6.5 and 6.7 , we have

$$
\begin{aligned}
\int_{0}^{1}\left(u_{s}^{\prime}\right)^{2} d x+h u_{s}^{2}(1)= & \int_{0}^{1} u_{s}^{2} f^{\prime}(\bar{u}) \bar{v} d x-\int_{0}^{1} f(\bar{u})\left(v_{0}^{0}+w_{0}^{0}+o(1)\right) u_{s} d x \\
& +\frac{h k}{\theta} u_{s}(1) \int_{0}^{1}(f(\bar{u})-f(1)) u_{s} d x+\frac{h k u_{s}(1) f(1)}{\theta} \int_{0}^{1} u_{s} d x \\
\leq & \varepsilon\left(\int_{0}^{1} u_{s}^{2} d x+h u_{s}^{2}(1)\right)+C / \varepsilon,
\end{aligned}
$$

for all small $s>0$. Thus, by the fact that the $\mathrm{H}^{1}(0,1)$-norm of $u_{s}$ is equivalent to the square root of the left-hand side of the above inequality, we have that the $\mathrm{H}^{1}(0,1)$-norm of $u_{s}$ is bounded uniformly for all small $s>0$. It follows that this is also true for the $C^{2+\alpha}[0,1]$-norm of $u_{s}$. Now, after passing to a subsequence, we have $u_{s} \rightarrow$ some $u_{0}^{0}$ in $C^{2}[0,1]$, where $u_{0}^{0}$ satisfies

$$
\left\{\begin{array}{l}
\left(u_{0}^{0}(x)\right)^{\prime \prime}+h u_{0}^{0}(1)=f(1)\left(v_{0}^{0}(x)+w_{0}^{0}(x)\right), \quad x \in(0,1), \\
\left(u_{0}^{0}\right)^{\prime}(0)=0=\left(u_{0}^{0}\right)^{\prime}(1)+h u_{0}^{0}(1), \\
\int_{0}^{1} u_{0}^{0}(x) d x=0 .
\end{array}\right.
$$

Since the solution (6.24) is unique, $u_{s} \rightarrow u_{0}^{0}$, without passing to a subsequence. This completes the proof of Lemma 6.8 .

LEMMA 6.9. For every $\lambda_{2}>\lambda_{0}^{0}$,

$$
\lim _{s \rightarrow 0^{+}} \frac{\phi_{2}(s)}{s}=-v_{0}^{0}, \quad \text { in } C^{2}[0,1] .
$$

Proof. Recall that we have chosen $\phi_{2}(s)$ such that

$$
\int_{0}^{1} \phi_{2}(s) d x=0 \text {. }
$$

Observe that

$$
\begin{cases}\left(\lambda_{1}\left(\frac{\phi_{2}}{s}\right)^{\prime}-\chi_{1} \phi^{\prime}(\bar{u}) \frac{\bar{u}^{\prime}}{s}\left(1+\phi_{2}\right)\right)^{\prime}+\frac{k f(\bar{u})-\theta}{s}\left(1+\phi_{2}\right)=0, & x \in(0,1), \\ \lambda_{1}\left(\frac{\phi_{2}}{s}\right)^{\prime}-\chi_{1} \phi^{\prime}(\bar{u}) \frac{\bar{u}^{\prime}}{s}\left(1+\phi_{2}\right)=0, & x=0,1 .\end{cases}
$$

Then the $C^{2+\alpha}$-norm of $\frac{\phi_{2}}{s}$ is bounded uniformly for small $s>0$ and hence, after passing to a subsequence, $\frac{\phi_{2}}{s} \rightarrow$ some $\phi_{2}^{0}$ in $C^{2}[0,1]$.

From the limiting equations that $\phi_{2}^{0}$ satisfies, it follows that

$$
\left(\phi_{2}^{0}\right)^{\prime}(x)=\frac{\chi_{1} \phi^{\prime}(1)}{\lambda_{1}}\left(u^{*}\right)^{\prime}(x)-\frac{1}{\lambda_{1}} F_{1}(x)=-\left(v_{0}^{0}\right)^{\prime}(x) \text { and } \int_{0}^{1} \phi_{2}^{0}(x) d x=0 .
$$


Thus $\phi_{2}^{0}(x)=-v_{0}^{0}(x)$.

LEMMA 6.10. For every $\lambda_{2}>\lambda_{0}^{0}$,

$$
\begin{aligned}
\lim _{s \rightarrow 0^{+}} \int_{0}^{1} k f^{\prime}(\bar{u}) \frac{u_{0}}{s^{2}} w_{0} W_{0} d x= & 2 k f^{\prime}(1) e^{-\chi_{2}^{0} \phi(1) / \lambda_{2}} \int_{0}^{1} u_{0}^{0}\left(v_{0}^{0}+w_{0}^{0}\right) d x \\
& -\frac{\chi_{2}^{0} \phi^{\prime}(1)}{\lambda_{2}} e^{-\chi_{2}^{0} \phi(1) / \lambda_{2}} \int_{0}^{1} u_{0}^{0} f_{1} d x .
\end{aligned}
$$

Proof. We start by studying $\lim _{s \rightarrow 0^{+}} \int_{0}^{1} k f^{\prime}(\bar{u}) \frac{u_{0}}{s^{2}} d x$.

Let $V_{0}=v_{0} e^{-\chi_{1} \phi(\bar{u}) / \lambda_{1}}$. Then

$$
\left\{\begin{array}{cl}
\lambda_{1}\left(e^{\chi_{1} \phi(\bar{u}) / \lambda_{1}} V_{0}^{\prime}\right)^{\prime}-\chi_{1}\left(\bar{v} \phi^{\prime}(\bar{u}) u_{0}^{\prime}+\phi^{\prime \prime}(\bar{u}) \bar{u}^{\prime} \bar{v} u_{0}\right)^{\prime} & \\
+k f^{\prime}(\bar{u}) \bar{v} u_{0}+(k f(\bar{u})-\theta) V_{0} e^{\chi_{1} \phi(\bar{u}) / \lambda_{1}}=0, & x \in(0,1), \\
\lambda_{1} e^{\chi_{1} \phi(\bar{u}) / \lambda_{1}} V_{0}^{\prime}-\chi_{1}\left(\bar{v} \phi^{\prime}(\bar{u}) u_{0}^{\prime}+\phi^{\prime \prime}(\bar{u}) \bar{u}^{\prime} \bar{v} u_{0}\right)=0, & x=0,1 .
\end{array}\right.
$$

Multiplying (6.27) by $\bar{V}$ and (6.6) by $V_{0}$, and then integrating by parts, we have

$$
\int_{0}^{1}\left[\chi_{1}\left(\bar{v} \phi^{\prime}(\bar{u}) u_{0}^{\prime}+\phi^{\prime \prime}(\bar{u}) \bar{u}^{\prime} \bar{v} u_{0}\right) \bar{V}^{\prime}+k f^{\prime}(\bar{u}) \bar{v} u_{0} \bar{V}\right] d x=0 .
$$

This, Lemmas 6.8 and 6.9 imply that as $s \rightarrow 0^{+}$,

$$
\begin{aligned}
\int_{0}^{1} k f^{\prime}(\bar{u}) \frac{u_{0}}{s^{2}}\left(1+\phi_{2}(s)\right)^{2} e^{-\chi_{1} \phi(\bar{u}) / \lambda_{1}} d x & \\
=-\int_{0}^{1} \chi_{1} \phi^{\prime \prime}(\bar{u}) \frac{\bar{u}^{\prime}}{s}\left(1+\phi_{2}(s)\right) \frac{u_{0}}{s} \frac{\bar{V}^{\prime}}{s} d x & -\chi_{1} \int_{0}^{1}\left(1+\phi_{2}(s)\right) \phi^{\prime}(\bar{u}) \frac{u_{0}^{\prime}}{s} \frac{\bar{V}^{\prime}}{s^{2}} d x \\
& \rightarrow \frac{\chi_{1}}{\lambda_{1}} \int_{0}^{1} \phi^{\prime}(1)\left(u_{0}^{0}\right)^{\prime} F_{1}(x) e^{-\chi_{1} \phi(1) / \lambda_{1}} d x ;
\end{aligned}
$$

$$
\begin{aligned}
\lim _{s \rightarrow 0^{+}} \int_{0}^{1} k f^{\prime}(\bar{u}) \frac{u_{0}}{s^{2}} d x= & \lim _{s \rightarrow 0^{+}} \int_{0}^{1} k f^{\prime}(\bar{u}) \frac{u_{0}}{s^{2}}\left(1+\phi_{2}(s)\right)^{2} e^{-\chi_{1}(\phi(\bar{u})-\phi(1)) / \lambda_{1}} d x \\
& -\lim _{s \rightarrow 0^{+}} \int_{0}^{1} k f^{\prime}(\bar{u}) \frac{u_{0}}{s} \cdot \frac{1}{s}\left[\left(1+\phi_{2}(s)\right)^{2} e^{-\chi_{1}(\phi(\bar{u})-\phi(1)) / \lambda_{1}}-1\right] d x \\
= & \frac{\chi_{1}}{\lambda_{1}} \phi^{\prime}(1) \int_{0}^{1}\left(u_{0}^{0}\right)^{\prime} F_{1}(x) d x+k f^{\prime}(1) \int_{0}^{1} u_{0}^{0}\left(2 v_{0}^{0}+\frac{\chi_{1}}{\lambda_{1}} \phi^{\prime}(1) u^{*}\right) d x .
\end{aligned}
$$

By this, Lemmas 6.3, 6.5, and 6.8, we have that as $s \rightarrow 0^{+}$,

$$
\begin{gathered}
\int_{0}^{1} k f^{\prime}(\bar{u}) \frac{u_{0}}{s^{2}} w_{0} W_{0} d x \\
=\int_{0}^{1} k f^{\prime}(\bar{u}) \frac{u_{0}}{s^{2}}\left(\int_{0}^{1} w_{0} d x+w_{0}^{0} s+o(s)\right)\left(\int_{0}^{1} W_{0} d x+W_{0}^{0} s+o(s)\right) d x \\
=\int_{0}^{1} k f^{\prime}(\bar{u}) \frac{u_{0}}{s^{2}} d x\left(\int_{0}^{1} w_{0} d x\right)\left(\int_{0}^{1} W_{0} d x\right)+\int_{0}^{1} k f^{\prime}(\bar{u}) \frac{u_{0}}{s}\left(W_{0}^{0} \int_{0}^{1} w_{0} d y\right. \\
\left.+w_{0}^{0} \int_{0}^{1} W_{0}^{0} d y\right) d x+o(1)
\end{gathered}
$$




$$
\begin{aligned}
& \rightarrow e^{-\chi_{2}^{0} \phi(1) / \lambda_{2}}\left[\frac{\chi_{1}}{\lambda_{1}} \phi^{\prime}(1) \int_{0}^{1}\left(u_{0}^{0}\right)^{\prime} F_{1}(x) d x+k f^{\prime}(1) \int_{0}^{1} u_{0}^{0}\left(2 v_{0}^{0}+\frac{\chi_{1}}{\lambda_{1}} \phi^{\prime}(1) u^{*}\right) d x\right] \\
& +\int_{0}^{1} k f^{\prime}(1) u_{0}^{0}\left(W_{0}^{0}+w_{0}^{0} e^{-\chi_{2}^{0} \phi(1) / \lambda_{2}}\right) d x \\
& =e^{-\chi_{2}^{0} \phi(1) / \lambda_{2}}\left[-\frac{\chi_{1}}{\lambda_{1}} \phi^{\prime}(1) \int_{0}^{1} u_{0}^{0} f_{1}(x) d x+k f^{\prime}(1) \int_{0}^{1} u_{0}^{0}\left(2 v_{0}^{0}+\frac{\chi_{1}}{\lambda_{1}} \phi^{\prime}(1) u^{*}\right) d x\right] \\
& +e^{-\chi_{2}^{0} \phi(1) / \lambda_{2}} \int_{0}^{1} k f^{\prime}(1) u_{0}^{0}\left(2 w_{0}^{0}-\frac{\chi_{2}^{0}}{\lambda_{2}} \phi^{\prime}(1) u^{*}\right) d x \\
& =e^{-\chi_{2}^{0} \phi(1) / \lambda_{2}}\left[2 k f^{\prime}(1) \int_{0}^{1} u_{0}^{0}\left(v_{0}^{0}+w_{0}^{0}\right) d x-\frac{\chi_{2}^{0} \phi^{\prime}(1)}{\lambda_{2}} \int_{0}^{1} u_{0}^{0} f_{1} d x\right] \text {, }
\end{aligned}
$$

where at the last step, we used the facts that $f_{1}=k f^{\prime}(1) u^{*}+\mu^{\prime}(0)$ and $\int_{0}^{1} u_{0}^{0} d x=0$.

LEMMA 6.11. For every $\lambda_{2}>\lambda_{0}^{0}$,

$$
\begin{aligned}
& \lim _{s \rightarrow 0^{+}} \int_{0}^{1} \frac{\chi\left(\lambda_{2}\right)}{s^{2}} u_{0} e^{\chi\left(\lambda_{2}\right) \phi(\bar{u}) / \lambda_{2}} \phi^{\prime}(\bar{u})\left(W_{0}^{\prime}\right)^{2} d x=0, \\
& \lim _{s \rightarrow 0^{+}} \int_{0}^{1} \frac{\chi\left(\lambda_{2}\right)}{\lambda_{2} s^{2}} u_{0} e^{\chi\left(\lambda_{2}\right) \phi(\bar{u}) / \lambda_{2}} \phi^{\prime}(\bar{u})(k f(\bar{u})-\theta) W_{0}^{2} d x \\
& \quad=\frac{\chi_{2}^{0} \phi^{\prime}(1)}{\lambda_{2}} e^{-\chi_{2}^{0} \phi(1) / \lambda_{2}} \int_{0}^{1} u_{0}^{0} f_{1} d x, \\
& \lim _{s \rightarrow 0^{+}} \int_{0}^{1} \frac{1}{s^{2}} \bar{u}^{\prime} \phi^{\prime}(\bar{u}) w_{0} W_{0}^{\prime} d x=-\frac{\phi^{\prime}(1)}{\lambda_{2}} e^{-\chi_{2}^{0} \phi(1) / \lambda_{2}} \int_{0}^{1}\left(u^{*}\right)^{\prime} F_{1}(x) d x>0 .
\end{aligned}
$$

Proof. By Lemmas 6.3 and 6.6, the first limit is equal to zero.

The second identity follows from Lemmas 6.3 and 6.8, the third from Lemmas 6.3 and 6.6 .

Proposition 6.12. For every $\lambda_{2}>\lambda_{0}^{0}, \lambda_{2} \neq \lambda_{1}$, there exists a positive constant $\delta>0$ such that $\left.\frac{d \chi_{2}(r)}{d r}\right|_{r=0} \geq \delta$ for all small $s>0$, and hence for all $\theta \in(0, k f(1))$ close to $k f(1)$.

Proof. By Lemmas 6.1, 6.10, and 6.11, we have

$$
\left.\lim _{s \rightarrow 0^{+}} \frac{d \chi_{2}(r)}{d r}\right|_{r=0}=\frac{2 k f^{\prime}(1) \lambda_{2} \int_{0}^{1} u_{0}^{0}\left(v_{0}^{0}+w_{0}^{0}\right) d x}{\phi^{\prime}(1) \int_{0}^{1}\left(u^{*}\right)^{\prime} F_{1}(x) d x} .
$$

Notice that $\left(u^{*}\right)^{\prime}(x)>0, F_{1}(x)<0$, for $x \in(0,1)$.

By (6.24), we have

$$
\int_{0}^{1} u_{0}^{0}\left(v_{0}^{0}+w_{0}^{0}\right) d x=-\left(\int_{0}^{1}\left(\left(u_{0}^{0}\right)^{\prime}\right)^{2} d x+h\left(u_{0}^{0}(1)\right)^{2}\right) / f(1)<0,
$$

unless $u_{0}^{0} \equiv 0$. If $u_{0}^{0} \equiv 0$, then (6.24) implies that $v_{0}^{0}+w_{0}^{0} \equiv 0$ and hence

$$
\left(\frac{1}{\lambda_{1}}-\frac{1}{\lambda_{2}}\right) F_{1}(x)+\phi^{\prime}(1)\left(\frac{\chi_{2}^{0}}{\lambda_{2}}-\frac{\chi_{1}}{\lambda_{1}}\right)\left(u^{*}\right)^{\prime}(x) \equiv 0 .
$$


This is impossible because $u^{*}$ is a quadratic polynomial while $F_{1}(x)$ is a cubic one. This completes the proof of Proposition 6.12.

Now we are ready to prove the main result of this section.

Theorem 6.13. For fixed $\lambda_{1}>0, \chi_{1} \geq 0$ and $\lambda_{2} \neq \lambda_{1}$ with $\lambda_{2}>\lambda_{0}^{0}\left(=2 \lambda_{1} k f^{\prime}(1) /\right.$ $\left.\left(2 k f^{\prime}(1)+21 \phi^{\prime}(1) \chi_{1}\right)\right)$, there exists a small $\delta_{0}>0$ such that for each fixed $\theta$ satisfying $k f(1)-\delta_{0}<\theta<k f(1)$, there exists a small $\varepsilon_{0}>0$ so that if $\chi\left(\lambda_{2}\right)<\chi_{2}<\chi\left(\lambda_{2}\right)+\varepsilon_{0}$, then the unique positive steady state of $(1.3)$ bifurcating from $(\bar{u}, \bar{v}, 0)$ is locally asymptotically stable in the $\mathrm{H}^{1}(0,1)$-topology.

Proof. We choose $\delta_{0}$ small enough so that if $k f(1)-\delta_{0}<\theta<k f(1)$, then $s$ is small enough so that Prop. 6.12 holds (recall $\mu(s)=k f(1)-\theta$ and $\mu^{\prime}(0)>0$ ). Furthermore, choose $\varepsilon_{0}>0$ small enough so that $\chi_{2}$ and $(u, v, w)$ mentioned above are given by $\chi_{2}=\chi_{2}(r),\left(u_{r}, v_{r}, w_{r}\right)=(\bar{u}+\tilde{u}(r), \bar{v}+\tilde{v}(r), \tilde{w}(r)), 0<r<\varepsilon_{1}$ (see Theorem 5.1). To show the stability of the steady state, we linearize $(3.1)$ at $\left(u_{r}, v_{r}, w_{r}\right)$ and study the following eigenvalue problem:

$$
\begin{cases}u^{\prime \prime}-f^{\prime}\left(u_{r}\right)\left(v_{r}+w_{r}\right) u-f\left(u_{r}\right)(v+w)=\eta u, & x \in(0,1), \\ u^{\prime}(0)=0=u^{\prime}(1)+h u(1), & \\ \left(B^{1}(u, v)\right)^{\prime}+k f^{\prime}\left(u_{r}\right) v_{r} u+\left(k f\left(u_{r}\right)-\theta\right) v=\eta v, & x \in(0,1), \\ B^{1}(u, v)=0, & x=0,1, \\ \left(B^{2}(u, w)\right)^{\prime}+k f^{\prime}\left(u_{r}\right) w_{r} u+\left(k f\left(u_{r}\right)-\theta\right) w=\eta w, & x \in(0,1), \\ B^{2}(u, w)=0, & x=0,1 .\end{cases}
$$

where

$$
\begin{aligned}
& B^{1}(u, v)=\lambda_{1} v^{\prime}-\chi_{1} u_{r}^{\prime} \phi^{\prime}\left(u_{r}\right) v-\chi_{1} \phi^{\prime}\left(u_{r}\right) v_{r} u^{\prime}-\chi_{1} \phi^{\prime \prime}\left(u_{r}\right) u_{r}^{\prime} v_{r} u \\
& B^{2}(u, w)=\lambda_{2} w^{\prime}-\chi_{2} u_{r}^{\prime} \phi^{\prime}\left(u_{r}\right) w-\chi_{2} \phi^{\prime}\left(u_{r}\right) w_{r} u^{\prime}-\chi_{2} \phi^{\prime \prime}\left(u_{r}\right) u_{r}^{\prime} w_{r} u
\end{aligned}
$$

Let $\eta(r)$ be the eigenvalue with the largest real part, and $(u, v, w)$ the corresponding eigenfunction normalized by $\|u\|_{L^{2}(0,1)}+\|v\|_{L^{2}(0,1)}+\|w\|_{L^{2}(0,1)}=2$. By the Principle of the Linearized Stability ([S, D]), the desired stability of $\left(u_{r}, v_{r}, w_{r}\right)$ will follow if we can show that $\operatorname{Re} \eta(r)<0$ for small $r>0$. Suppose there exists a sequence of $r \rightarrow 0^{+}$such that $\operatorname{Re} \eta(r) \geq 0$. It is easy to show that as $r \rightarrow 0^{+}$, (a) $\eta(r)$ is bounded, (b) $(u, v, w)$ is bounded in $C^{2+\alpha}[0,1]$. Then after passing to a subsequence, we have $\eta \rightarrow$ some $\eta_{0}$ with $\operatorname{Re} \eta_{0} \geq 0$, and $(u, v, w) \rightarrow\left(u^{0}, v^{0}, w^{0}\right)$ in $C^{2}[0,1]$, where $\left(u_{0}, v_{0}, w_{0}\right)$ satisfies (4.3) with $\eta=\eta_{0}$ and $\chi_{2}=\chi_{2}(0)\left(=\chi\left(\lambda_{2}\right)\right)$. If $w^{0}=0$, then $\left(u^{0}, v^{0}\right) \neq(0,0)$ and since $\theta$ is close to $k f(1)$, we have the stability of $(\bar{u}, \bar{v})$ with respect to the single species dynamics which implies $\operatorname{Re} \eta_{0}<0$. So $w^{0} \neq 0$. But since $\eta^{*}\left(\lambda_{2}, \chi\left(\lambda_{2}\right)\right)=0, \eta_{0}=0$. Thus $\left(u^{0}, v^{0}, w^{0}\right)= \pm\left(u_{0}, v_{0}, w_{0}\right)$. Now multiplying $(u, v, w)$ by \pm 1 , we have that

$$
(u, v, w) \rightarrow\left(u_{0}, v_{0}, w_{0}\right) \quad \text { in } C^{2}[0,1] \text { as } r \rightarrow 0 .
$$

Differentiating with respect to $r$ the $w$-equation in $(3.1)$ (with $(u, v, w)=\left(u_{r}, v_{r}, w_{r}\right)$ ), we obtain

$$
\begin{cases}\left(B^{3}\left(u_{r}, w_{r}\right)\right)^{\prime}+k f^{\prime}\left(u_{r}\right) \dot{u}_{r} w_{r}+\left(k f\left(u_{r}\right)-\theta\right) \dot{w}_{r}=0, & x \in(0,1), \\ B^{3}\left(u_{r}, w_{r}\right)=0, & x=0,1,\end{cases}
$$


where $\dot{u}_{r}=\frac{d}{d r} u_{r}=u_{0}+o(1), \dot{w}_{r}=\frac{d}{d r} w_{r}=w_{0}+o(1)$, and

$$
\begin{aligned}
B^{3}\left(u_{r}, w_{r}\right)=\lambda_{2} \dot{w}_{r}^{\prime}-\frac{d \chi_{2}(r)}{d r}\left(\phi^{\prime}\left(u_{r}\right) u_{r}^{\prime} w_{r}\right)-\chi_{2}(r) \phi^{\prime \prime}\left(u_{r}\right) \dot{u}_{r} u_{r}^{\prime} w_{r} \\
\\
-\chi_{2}(r) \phi^{\prime}\left(u_{r}\right) \dot{u}_{r}^{\prime} w_{r}-\chi_{2}(r) \phi^{\prime}\left(u_{r}\right) u_{r}^{\prime} \dot{w}_{r} .
\end{aligned}
$$

Define $\dot{\mathscr{W}}_{r}=\dot{\mathrm{w}}_{r} e^{-\chi_{2}(r) \phi\left(u_{r}\right) / \lambda_{2}}$. Then

$$
\begin{aligned}
B^{3}\left(u_{r}, w_{r}\right)=\lambda_{2} e^{\chi_{2}(r) \phi\left(u_{r}\right) / \lambda_{2}} \dot{W}_{r}^{\prime}-\frac{d \chi_{2}(r)}{d r}( & \left.\phi^{\prime}\left(u_{r}\right) u_{r}^{\prime} w_{r}\right) \\
& -\chi_{2}(r)\left(\phi^{\prime \prime}\left(u_{r}\right) \dot{u}_{r} u_{r}^{\prime} w_{r}+\phi^{\prime}\left(u_{r}\right) u_{r}^{\prime} \dot{w}_{r}\right) .
\end{aligned}
$$

Let $W=w e^{-\chi_{2}(r) \phi\left(u_{r}\right) / \lambda_{2}}$. Then

$$
B^{2}(u, w)=\lambda_{2} e^{\chi_{2}(r) \phi\left(u_{r}\right) / \lambda_{2}} W^{\prime}-\chi_{2}\left(\phi^{\prime}\left(u_{r}\right) w_{r} u^{\prime}+\phi^{\prime \prime}\left(u_{r}\right) u_{r}^{\prime} w_{r} u\right)
$$

Now, multiplying the $w$-equation in (6.28) by $\dot{W}_{r}$ and (6.30) by $W$, and integrating by parts, we have

$$
\begin{array}{r}
\eta(r) \int_{0}^{1} w \dot{W}_{r} d x=\int_{0}^{1} k f^{\prime}\left(u_{r}\right) w_{r}\left(u \dot{W}_{r}-\dot{u}_{r} W\right) d x-\frac{d \chi_{2}(r)}{d r} \int_{0}^{1} \phi^{\prime}\left(u_{r}\right) u_{r}^{\prime} w_{r} W^{\prime} d x \\
+\chi_{2}(r) \int_{0}^{1} \phi^{\prime}\left(u_{r}\right)\left(w_{r} u^{\prime} \dot{W}_{r}^{\prime}-\dot{u}_{r}^{\prime} w_{r} W^{\prime}\right) d x+\chi_{2}(r) \int_{0}^{1} \phi^{\prime \prime}\left(u_{r}\right)\left(u_{r}^{\prime} w_{r} u \dot{W}_{r}^{\prime}\right. \\
\left.-\dot{u}_{r} u_{r}^{\prime} w_{r} W^{\prime}\right) d x=I_{1}-I_{2}+I_{3}+I_{4} .
\end{array}
$$

From (6.29) and Prop. 6.12, it follows that as $r \rightarrow 0$,

$$
\begin{gathered}
I_{1}=o(r) ; \\
I_{2}=\left(\left.\frac{d \chi_{2}(r)}{d r}\right|_{r=0}+o(1)\right) \int_{0}^{1}\left(\phi^{\prime}(\bar{u})+o(1)\right)\left(\bar{u}^{\prime}+o(1)\right) r\left(w_{0}+o(1)\right) W_{0} d x \\
\left.=\frac{\delta r}{2} \int_{0}^{1} \bar{u}^{\prime} \phi^{\prime}(\bar{u}) w_{0} W_{0} d x+o(r)=\text { (positive const. }\right) r+o(r)
\end{gathered}
$$

(recall that $w_{0}$ is close to 1 when $\theta$ is close to $k f(1)$ - see Lemma 6.3);

$$
\begin{gathered}
I_{3}=o(r) ; \quad I_{4}=o(r) \\
\int_{0}^{1} w \dot{W}_{r} d x=\int_{0}^{1}\left(w_{0}+o(1)\right)\left(W_{0}+o(1)\right) d x=\text { positive const. }+o(r) .
\end{gathered}
$$

Thus $\operatorname{Re} \eta(r)<0$ for small $r>0$, a contradiction!

This completes the proof of Theorem 6.13.

Acknowledgments. This work is supported in part by the National Natural Science Foundation of China, the Beijing Natural Science Foundation, and the National Science Foundation of the USA. XW is grateful to the Mathematics Departments of Peking University and Capital Normal University, especially Professors K. C. Chang, Z. Y. Li, and Q. X. Ye for their supporting his visits to Beijing. 


\section{REFERENCES}

[BB] J. Blat and K. Brown, Global bifurcation of positive solutions in some systems of elliptic equations, SIAM J. Math. Anal. 17, 1339-1353 (1986)

[CR] M. Crandall and P. Rabinowitz, Bifurcation, perturbation of simple eigenvalues and linearized stability, Arch. Rational Mech. Anal. 52, 161-180 (1973)

[D] A.-K. Drangeid, The principle of linearized stability for quasilinear parabolic evolution equations, Nonlinear Anal. 13, 1091-1113 (1989)

[DHMP] J. Dockery, V. Hutson, K. Mischaikow, and M. Pernarowski, The evolution of slow dispersal rates: A reaction diffusion model, J. Math. Biol. 37 61-83 (1998)

[LAK] D. Lauffenburger, R. Aris, and K. Keller, Effects of cell motility and chemotaxis on microbial population growth, J. Biophys. Soc. 40, 209-219 (1982)

[LC] D. Lauffenburger and P. Calcano, Competition between two microbial populations in a nonmixed environment: Effect of cell random motility, Biotech. and Bioengrg. 25, 2103-2125 (1983)

[R] P. Rabinowitz, Some global results for nonlinear eigenvalue problems, J. Functional Analysis 7, 487-513 (1971)

[S] G. Simonett, Center manifolds for quasilinear reaction-diffusion systems, Differential Integral Equations 8, 753-796 (1995)

[W] X.-F. Wang, Qualitative behavior of solutions of chemotactic diffusion systems: Effects of motility and chemotaxis and dynamics, SIAM J. Math. Anal. 31 535-560 (2000)

[Z] B. Zeng, Steady state solutions to a model for chemotaxis, Math. Appl. 3, 78-83 (1990) (in Chinese) 